\title{
Phytoplankton community and physical-chemical characteristics of water in the public reservoir of Cruzeta, $\mathrm{RN}$, Brazil
}

\author{
Chellappa, $N T^{\mathrm{a} *}{ }^{*}, B o r b a, J M .^{\mathrm{a}}$ and Rocha, $O .^{\mathrm{b}}$ \\ aPós-graduação em Bioecologia aquática, Departamento de Oceanografia e Limnologia, \\ Universidade Federal do Rio Grande do Norte - UFRN, \\ Av. Via Costeira, CEP 59014-100, Natal, RN, Brazil \\ bDepartamento de Ecologia e Biologia Evolutiva, Universidade Federal de São Carlos - UFSCar, \\ Rodovia Washington Luis, Km 235, CEP 13564-905, São Carlos, SP, Brazil \\ *e-mail: chellappa@dol.ufrn.br \\ Received September 21, 2006 - Accepted March 9, 2007 - Distributed August 31, 2008
}

(With 8 figures)

\begin{abstract}
The Phytoplankton community and the abiotic factors of the Cruzeta reservoir were studied at three depths, surface, middle $(2 \mathrm{~m})$ and bottom, from September, 2004 to June, 2005, in order to characterize the environment and assess the possible factors that influence the compositional change of phytoplankton. Ninety species belonging to 6 classes (Chlorophyceae, Bacillariophyceae, Cyanophyceae, Dinophyceae, Chrysophyceae and Euglenophyceae) were identified with 66 and 80 taxonomic units in the dry and rainy season. The most representative class in terms of species richness was Chlorophyceae and dominated by Scendesmus quadricauda, Oocystis sp. and Chlorella sp. The group cyanobacteria were represented by 18 species of diverse morphological characteristics and the dominance of Cylindrospermopsis raciborskii in September 2004. The other major group, Bacillariophyceae is represented by 21 species with the predominance of Aulacoseira granulata in mid-column and bottom waters. The other dominant species was Phacus acuminatus of Euglenophyceae. The species diversity and evenness indices were high, moderate and low in relation to the three hydroperiod registered during the 2004-2005 annual cycle. The reservoir exhibits high electrical conductivity (290-550 $\left.\mu \mathrm{S} . \mathrm{cm}^{-1}\right)$, alkaline $\mathrm{pH}$ (7.3-9.4), mean temperature of $28{ }^{\circ} \mathrm{C}$, varying concentrations of dissolved oxygen (3.29-7.6 mg. $\mathrm{L}^{-1}$ ) and the greatest concentration of nutrients at the bottom (orthophosphate, 0.22-0.62 mg. $\mathrm{L}^{-1}$ ) with the general tendency of oligo-mesotrophic status during sampling periods. The chlorophyll $a$ fluctuated to a minimum of $1.34 \mu \mathrm{g} . \mathrm{L}^{-1}$ at the bottom in April, 2005 and a maximum of $14.3 \mu \mathrm{g} . \mathrm{L}^{-1}$ in mid-column water in September, 2004. The reservoir is characteristically an oligo-mesotrophic environment.
\end{abstract}

Keywords: Cruzeta RN reservoir, hydroperiod, phytoplankton, chlorophyll $a$, nutrients.

\section{Comunidade fitoplanctônica e características físico-químicas do açude público de Cruzeta, Rio Grande do Norte, BR}

\begin{abstract}
Resumo
A comunidade fitoplanctônica e os fatores abióticos do açude Cruzeta foram estudados em três profundidades, superfície, meio (2 m) e fundo, de setembro, 2004 a junho, 2005, com o intuito de caracterizar e avaliar os possíveis fatores que influenciam as mudanças composicionais do fitoplâncton. Noventa espécies pertencentes a 06 classes (Chlorophyceae, Bacillariophyceae, Cyanophyceae, Dinophyceae, Chrysophyceae e Euglenophyceae) foram identificadas com 66 e 80 unidades taxonômicas nas estações de seca e de chuvas. A riqueza de espécies foi classe Chlorophyceae, com dominância de Scenedesmus quadriculata, Oocystis sp. e Chlorella sp. O grupo das Cyanophyceae foi representado por 18 espécies de características diversas com dominância de Cylindrospermopsis raciborskii em Setembro 2004. Em seguida, foi encontrado o grupo das Bacillariophyceae, representado por 21 espécies com a predominância de Aulacoseira granulata no fundo d'água. Outra espécie dominante foi Phacus acuminatus das Euglenophyceae. Os índices de diversidade e similaridade foram classificados como alto, moderado e baixo em relação aos três hidroperíodos registrados, durante o ciclo anual de 2004-2005. O reservatório exibiu alta condutividade elétrica (290-550 $\mu$ S.cm $\left.{ }^{-1}\right)$, $\mathrm{pH}$ alcalino (7.3-9.4), temperatura média de $28^{\circ} \mathrm{C}$, concentrações de oxigênio dissolvido variando entre $3.29 \mathrm{mg} . \mathrm{L}^{-1} \mathrm{a}$ $7.6 \mathrm{mg} . \mathrm{L}^{-1}$ e maior concentração de nutrientes no fundo do reservatório (ortofosfato, 0.22-0.62 mg. $\mathrm{L}^{-1}$ ), com tendência geral ao estado oligo-mesotrófico durante o período de amostragens. A clorofila a flutuou de um mínimo de $1.34 \mu \mathrm{g} . \mathrm{L}^{-1}$ no fundo em Abril, 2005 e um máximo de $14.3 \mu \mathrm{g} . \mathrm{L}^{-1}$ no meio da coluna d'água em Setembro, 2004. O reservatório é um ambiente oligo-mesotrófico característico.
\end{abstract}

Palavras-chave: açude Cruzeta RN, hidroperíodo, fitoplâncton, clorofila $a$, nutrientes. 


\section{Introduction}

The Phytoplankton biomass in reservoirs depends on various interrelated physical, chemical and biological factors (Kimmel et al., 1999) and is subject to the action of the pulses produced in the system that may be of natural origin, such as precipitation, wind and the influx of the river, or anthropogenic (Tundisi et al., 1999). Therefore, the phytoplanktonic communities display a potential for change that may lead to the substitution, removal or addition of species. Alterations in species richness are mainly due to the variability of abiotic factors, such as climactic tendencies or short-term climatic variations (Cody, 1996). Freshwater phytoplankton ecology studies in Brazil include reservoirs, natural lakes coastal lagoons, floodplain lakes, rivers and fish ponds. Nearly $75 \%$ of the studies come from South and Southeastern region of Brazil based on both descriptive and experimental approaches (Barbosa et al., 1995). The most important contributions are based on the general phytoplankton structure, diversity to cyanobacterial dominance and their toxin producing capacity associated with eutrophic conditions (Azevedo et al., 1994; Bicudo et al., 1999; Huszar et al., 2000). By comparison, relatively few studies have been conducted in the Northeast (Henry, 1999). Although limited in number of publications, the investigations on the ecology of phytoplankton that have been carried out over a period of 15 years indicate the threat posed by the recurrence of two cyanobacteria, Microcystis aeruginosa and Cylindrospermopsis raciborskii (Bouvy et al., 2000 and 2003; Chellappa, 1990; Chellappa and Costa, 2003; Chellappa et al., 1996). The synoptic studies researched in Pernambuco state, indicate that the high temperature, alkaline $\mathrm{pH}$, long water retention time and eutrophic to hypereutrophic status and luxury uptake of nitrogen and phosphorus by dominant cyanobacterial species determined phytoplankton composition in 39 Brazilian northeast reservoirs. The research also revealed the fast spreading nature of invasive cyanobacterium, Cylindrospermopsis raciborskii (Bouvy et al., 2000; Bouvy et al., 2003).

In Rio Grande do Norte, studies performed with limnetic phytoplankton were intensified in the 1990s. Chellappa et al. (1996) assessed semi-arid ecosystems in Rio Grande do Norte, comparing the phytoplankton composition of oligotrophic and eutrophic lakes and observing the diversity of species related to the trophic status of Lakes Urubu and Extremoz. Chellapa et al. (2000) studied the phytoplankton community of São Paulo do Potengi reservoir, a salinized fresh water, where the spatial-temporal effect of eutrophication was verified, highlighting the alternating dominance between Aulocoseira granulata (Bacillariophyceae) and Spirulina platensis (Cyanobacteria). Studies performed at Armando Ribeiro Gonçalves reservoir in Açu, RN and at Marechal Dutra (Gargalheiras) reservoir in Acari, RN (Chellappa et al., 2000, Chellappa and Costa, 2003) also demonstrated a high degree of water eutrophication, which stimulated the dominance of toxic cyanobacteria. Medeiros (2005) studied the phytoplankton composition of the public reservoir of Cruzeta, $\mathrm{RN}$, in which it was detected the presence of potentially toxic cyanobacteria, such as Anabaena circinalis and Cylindrospermopsis raciborskii.

There are two ecological hypotheses successfully tried by experimental basis to determine phytoplankton growth and biomass production: trophic cascade theory, which indicates how a strong reduction of the fish stock leads to marked increase in the zooplankton community. Many phytoplanktivorous zooplankton graze down phytoplankton biomass to a low level (Meijer et al., 1994; Nogueira et al., 2005). The second hypothesis is based on bottom-up theory where the nutrients from sediment suspension result in positive feedback to stimulate bloom formation and high phytoplankton biomass (Carpenter et al., 1985; Pinto-Coelho et al., 2005). Shallow water reservoirs of northeast Brazil are mostly governed by drought polygon where the low annual rainfall and high evaporation rate coupled with reservoir draw down (flushing) determine the phytoplankton structure (Bouvy et al., 2003; Chellappa and Costa, 2003). The purpose of the present study was to characterize the phytoplankton community of the public reservoir of Cruzeta in a vertical profile during a drought-rainfall cycle in 2004-2005 and to find out which factor or factors determine community structure, diversity and chlorophyll biomass.

\section{Material and Methods}

The Public Reservoir of Cruzeta is located in the municipality of Cruzeta in the Western Seridó region of the state of Rio Grande do Norte (geographic coordinates $06^{\circ} 24^{\prime} 42^{\prime \prime} \mathrm{S}$ and $36^{\circ} 47^{\prime} 23^{\prime \prime} \mathrm{W}$ ) (Figure 1).

It is a shallow reservoir of great importance for the municipality, since it provides water for irrigation and represents its only water source. The morphometric characteristics of the reservoir are: water holding capacity $35,000,000 \mathrm{~m}^{3}$, discharge rate $0.174 \mathrm{~m}^{3} / \mathrm{s}$, volume of water during the study period $26,010,000 \mathrm{~m}^{3}$ with a theoretical renewal time of around 180 days, maximum depth $11 \mathrm{~m}$ and mean depth of $4.5 \mathrm{~m}$.

Samples were collected monthly from September, 2004 to June, 2005 at a fixed collection station with a Van Dorn bottle $(5 \mathrm{~L})$ and at three depths: surface $(0 \mathrm{~m})$, $2 \mathrm{~m}$ and bottom $(6 \mathrm{~m})$. The following parameters were measured: $\mathrm{pH}$, temperature, electrical conductivity and dissolved oxygen (WTW Multiparameter Multi 340i). Nutrient analyses such as, nitrate (Golterman et al., 1978), orthophosphate (APHA, 1985), ammonium (Golterman et al., 1978) and chlorophyll- $a$ concentrations (Golterman et al., 1978) were conducted in the laboratory. For qualitative analysis of phytoplankton, the samples were preserved in acetic Lugol solution and subsequently examined under a Taimin TM800 optical microscope. The updated Identification of Freshwater Algae Manual was used predominantly for identification 

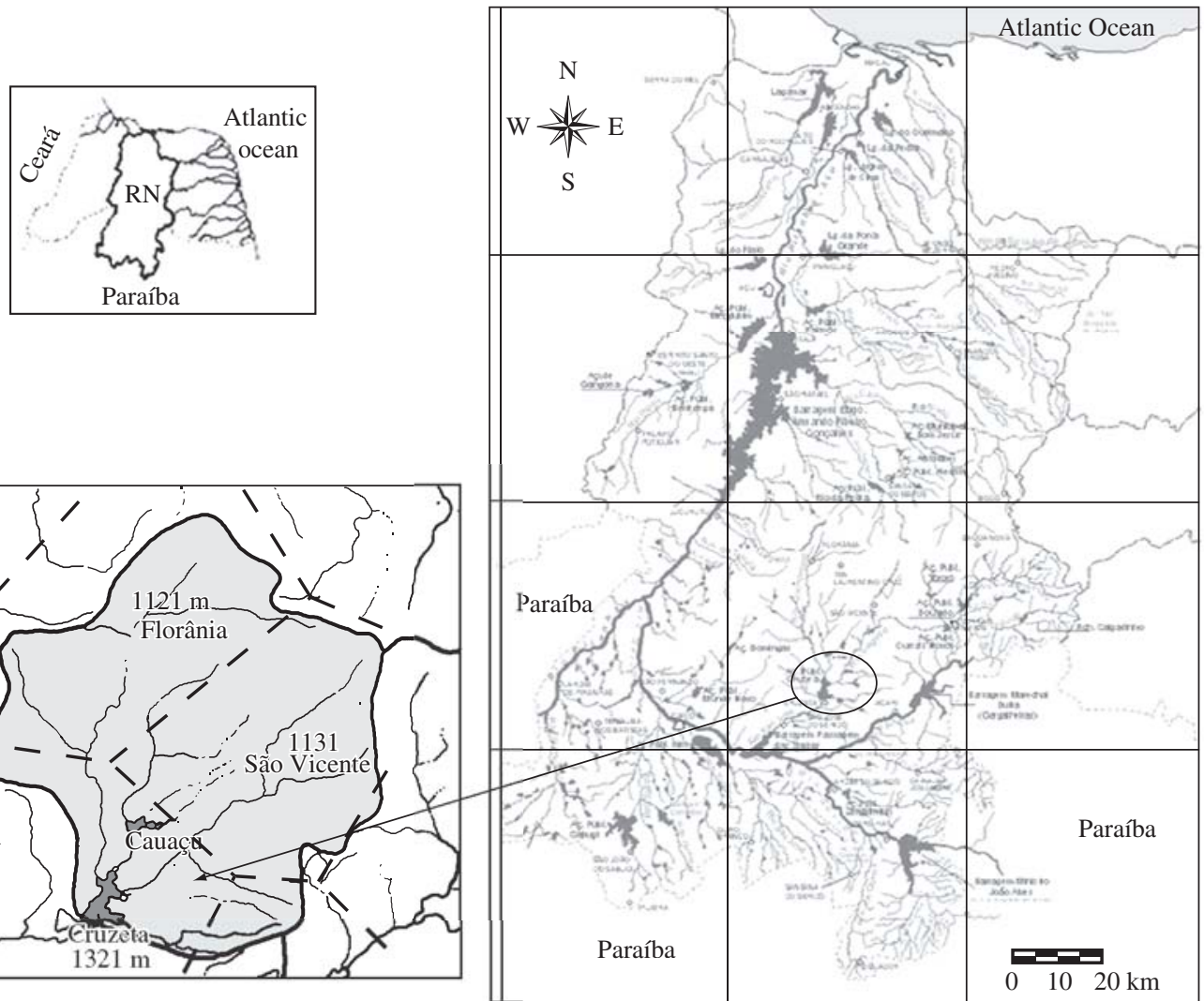

Figure 1. Hydrographic basin of Piranhas-Açu river and the location of Cruzeta reservoir, RN.

of species (Wehr and Sheath, 2003). The quantitative measurements of phytoplankton individuals were done using the sedimentation technique and counting with the help of a Sedgwick Rafter chamber. The Pearson correlation test was used to assess relations among groups and species of phytoplankton with environmental variables using the Statistics 6.0 program.

\section{Results}

The recorded total annual rainfall (mean of 20042005 was $328 \mathrm{~mm}$ ) is typical of the semiarid northeast region reaching a peak in March 2005 and minimum in the dry period (September to December) (Figure 2). Table 1 presents the data related to optical characteristics (euphotic zone and turbidity levels). Mean transparency during the dry and rainy periods was 0.43 and $0.57 \mathrm{~m}$, respectively, but more light penetrated the water column during the dry period compared to the wet period in response to the clear and turbid nature of the shallow water in the Cruzeta reservoir (Table 1).

The mean annual water temperature was $27^{\circ} \mathrm{C}$, with a maximum surface value $\left(30.05^{\circ} \mathrm{C}\right)$ in February and a minimum bottom value $\left(25.2{ }^{\circ} \mathrm{C}\right)$ in June, 2005. This month showed the lowest temperatures across the water column. The reduced depth of the Cruzeta reservoir determined the isothermal pattern and total mix-

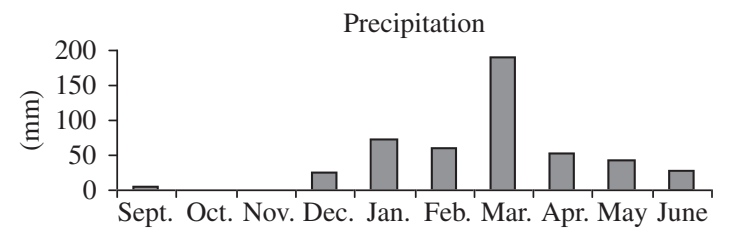

Figure 2. Monthly rainfall data of Cruzeta, RN reservoir (Sept/04 to June/05).

ing regime during the study period. The concentration of dissolved oxygen in the Cruzeta reservoir oscillated between $3.17 \mathrm{mg} . \mathrm{L}^{-1}$ at the $2 \mathrm{~m}$ depth in December and $7.60 \mathrm{mg} . \mathrm{L}^{-1}$ on the surface in June (Figure 3).

Electrical conductivity was elevated, a fact often observed in reservoirs in the semi-arid regions of northeastern Brazil, indicating a good buffered system. The lowest electrical conductivity values were observed at the onset of the drought in September $\left(291 \mu \mathrm{S} . \mathrm{cm}^{-1}\right)$, and the highest conductivity value was registered in the middle of the water column in April $\left(548 \mu \mathrm{S} . \mathrm{cm}^{-1}\right)$. The mean was $330 \mu \mathrm{S} . \mathrm{cm}^{-1}$ for the dry period and $435 \mu \mathrm{S} . \mathrm{cm}^{-1}$ for the rainy season. The $\mathrm{pH}$ had a mean value of 9.06 in the dry season and 8.70 in the rainy period, always alkaline, which is evidence of a good buffering system. The lowest value (7.39) was observed in the middle of the water 

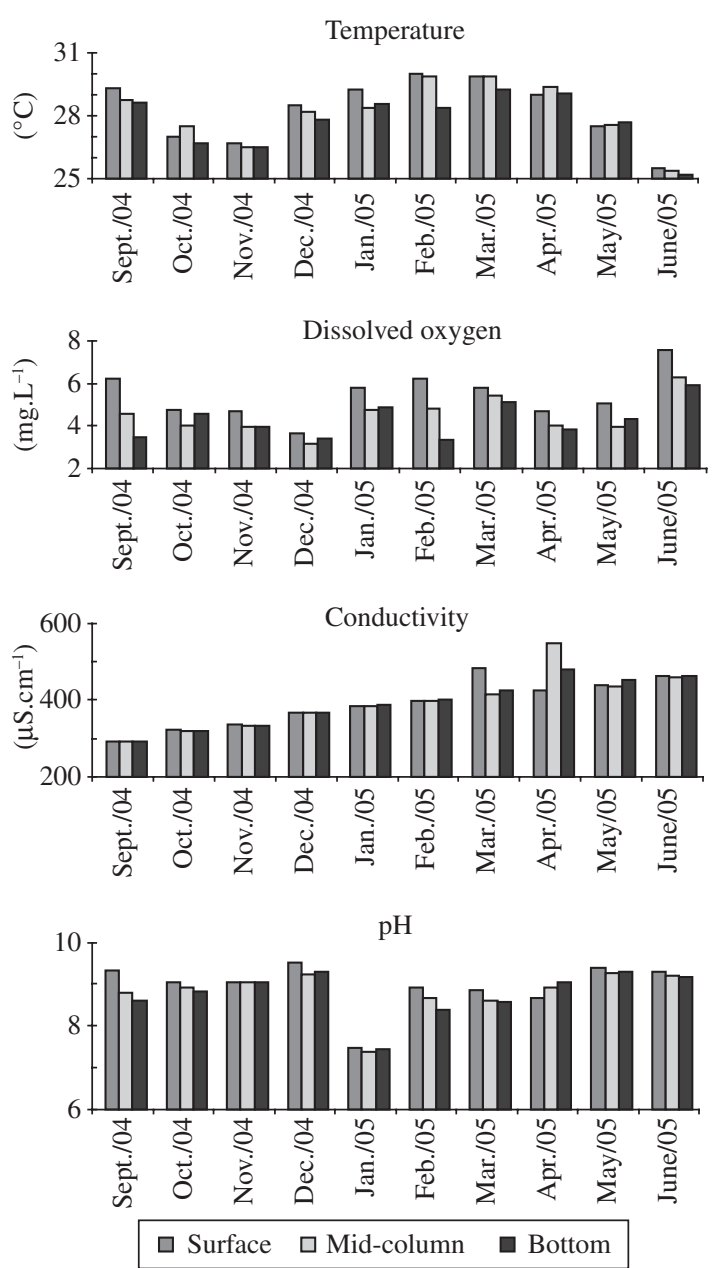

Figure 3. Seasonal variations of Temperature, Dissolved Oxygen, Conductivity and $\mathrm{pH}$ of water samples of Cruzeta, RN reservoir.

column in January and the highest $\mathrm{pH}$ value (9.53) was recorded on the surface in December (Figure 3).

The variations in orthophosphate, nitrate and ammonium are presented in Figure 4. An absolute nutrient value of nitrate and phosphate when transformed into a N/P ratio demonstrated low nitrogen to phosphorus status and the one based on the Redfield ratio gave a very insignificant value frequently around 0 or below 10 . Cyanobacterial abundance was incompatible to low N/P ratio in all three profiles.

Chlorophyll- $a$ concentrations varied from $1.34 \mu \mathrm{g} . \mathrm{L}^{-1}$ at the bottom in April to $14.26 \mu \mathrm{g} . \mathrm{L}^{-1}$ at middle depth $(2 \mathrm{~m})$ in September, due to the presence and dominance of Cylindrospermopsis raciborskii in the reservoir. The greatest concentrations of chlorophyll- $a$ were generally observed at mid-depth (Figure 5). Mean chlorophyll concentration was greater during the dry season. In this study, 95 taxa of algae were identified belonging to 6 classes (Chlorophyceae, Bacillariophyceae, Cyanophyceae,
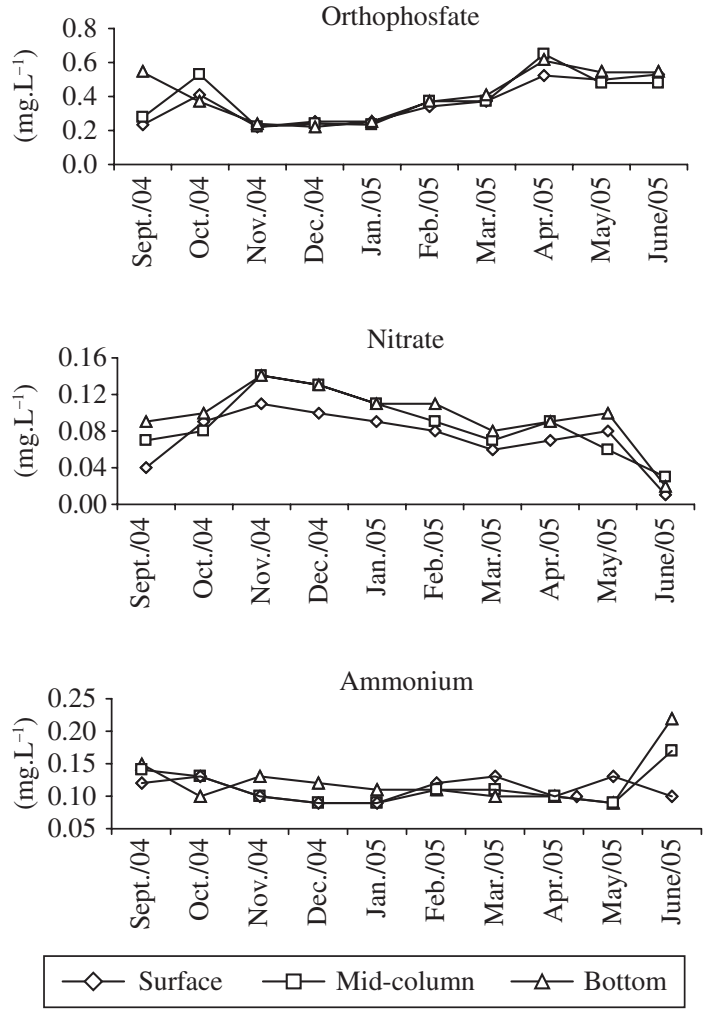

Figure 4. Seasonal variation in inorganic nutrients concentration of water samples of Cruzeta, $\mathrm{RN}$ reservoir.

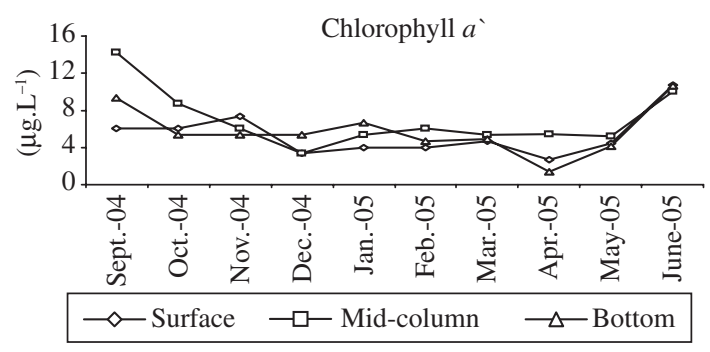

Figure 5. Seasonal variation of chlorophyll a concentrations in different depth profiles of Cruzeta, $\mathrm{RN}$ reservoir.

Table 1. Secchi disc measurement, Euphotic zone and depth profile of Cruzeta, RN reservoir during the study period (September/04 to June/05).

\begin{tabular}{lccccc}
\hline \multicolumn{1}{c}{ Date } & $\begin{array}{c}\mathbf{Z}_{\text {DS }} \\
(\mathbf{m})\end{array}$ & $\begin{array}{c}\mathbf{Z}_{\max } \\
(\mathbf{m})\end{array}$ & $\begin{array}{c}\mathbf{Z}_{\text {euf }} \\
(\mathbf{m})\end{array}$ & $\mathbf{Z}_{\max } / \mathbf{Z}_{\text {euf }}$ & $\begin{array}{c}\mathbf{K} \\
\left(\mathbf{m}^{-1}\right)\end{array}$ \\
\hline Sept./04 & 0.48 & 3.5 & 1.44 & 2.17 & 18.75 \\
Oct./04 & 0.44 & 3.2 & 1.32 & 2.10 & 20.45 \\
Nov./04 & 0.42 & 3.0 & 1.26 & 2.97 & 21.43 \\
Dec./04 & 0.40 & 3.5 & 1.20 & 2.75 & 22.50 \\
Jan./05 & 0.52 & 4.2 & 1.56 & 1.56 & 17.31 \\
Feb./05 & 0.68 & 4.0 & 2.04 & 1.96 & 13.24 \\
Mar./05 & 0.60 & 6.5 & 1.20 & 1.50 & 15.00 \\
Apr.05 & 0.52 & 5.0 & 1.25 & 1.56 & 17.31 \\
May/05 & 0.53 & 4.5 & 1.39 & 2.20 & 16.98 \\
Jun./05 & 0.54 & 4.0 & 1.42 & 1.85 & 16.67 \\
\hline
\end{tabular}


Dinophyceae, Chrysophyceae and Euglenophyceae), with a total of 66 in the dry period and 80 in the rainy season (Tables 2, 3 and 4). The most representative class in terms of species richness was Chlorophyceae
(42 species, with 26 in the dry period and 36 in the rainy season), followed immediately by Bacillariophyceae (28 species, with 18 in the dry period and 23 in the rainy season) (Figures 6, 7 and 8). In the vertical profiles,

Table 2. Phytoplankton species and relative abundance distributed in the surface water of Cruzeta/RN Reservoir during the annual cycle of 2004-2005.

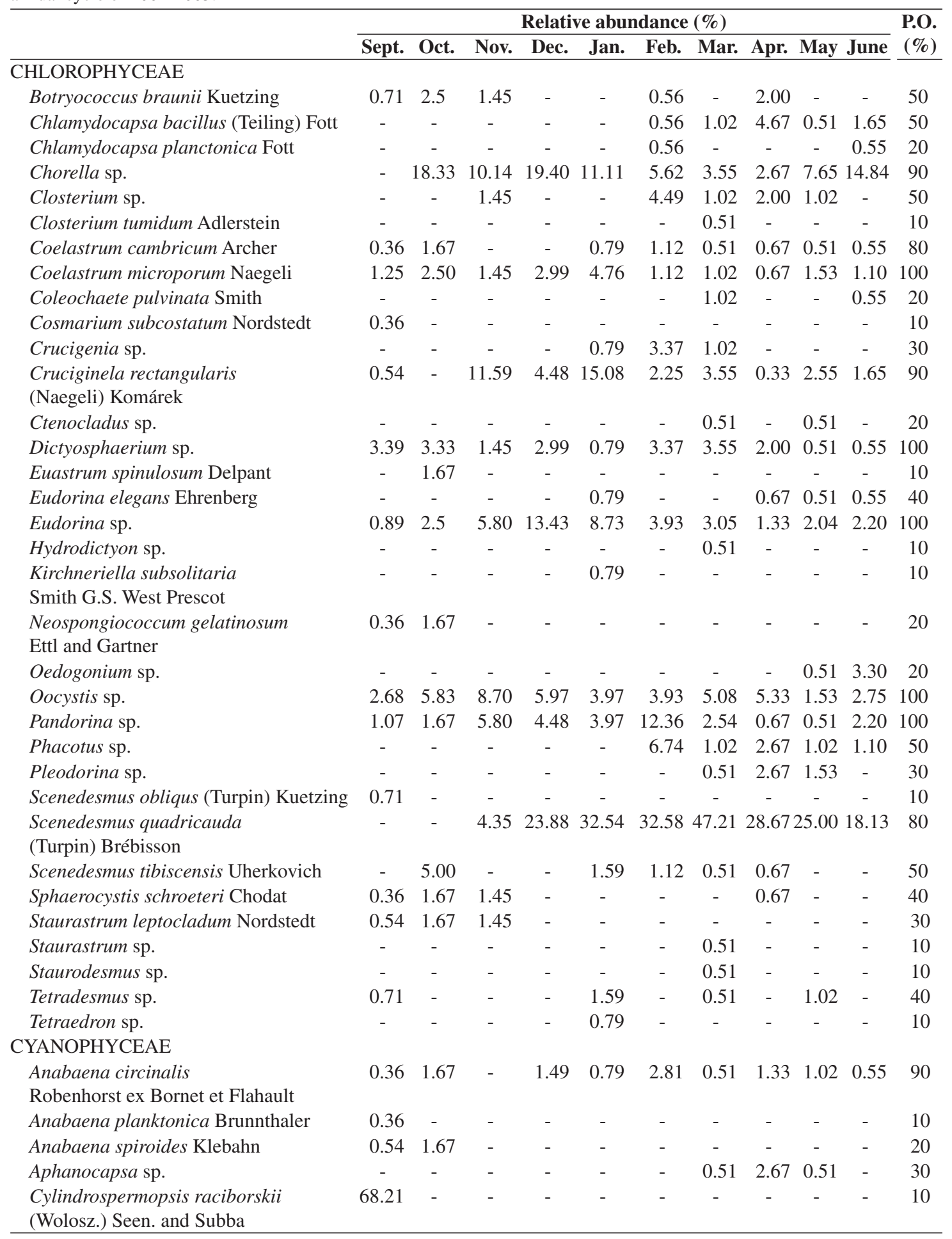


Table 2. Continued...

\begin{tabular}{|c|c|c|c|c|c|c|c|c|c|c|c|}
\hline & \multicolumn{10}{|c|}{ Relative abundance (\%) } & \multirow{2}{*}{$\begin{array}{l}\text { P.O. } \\
(\%)\end{array}$} \\
\hline & Sept. & Oct. & Nov. & Dec. & Jan. & Feb. & Mar. & Apr. & May & June & \\
\hline Eucapsis sp. & - & - & - & - & 0.79 & 1.69 & - & 0.67 & 0.51 & 0.55 & 50 \\
\hline Gloeothece sp. & - & - & - & - & - & - & - & - & 0.51 & - & 10 \\
\hline Lyngbya sp. & 1.43 & - & - & - & - & - & 1.02 & 0.67 & 0.51 & 0.55 & 50 \\
\hline Oscillatoria limosa Ag. & 8.93 & 1.67 & 2.9 & - & - & - & - & - & 0.51 & 0.55 & 50 \\
\hline $\begin{array}{l}\text { Planktolyngbya contorta } \\
\text { (Smith) Echdrateva }\end{array}$ & 0.36 & 1.67 & - & - & - & - & 0.51 & - & 0.51 & - & 40 \\
\hline Planktothrix sp. & - & - & 1.45 & - & - & - & 0.51 & 0.67 & 2.55 & - & 40 \\
\hline Pseudoanabaena catenata & 0.54 & - & - & - & - & - & 1.52 & 3.33 & 4.08 & 1.65 & 50 \\
\hline Raphidiopsis curvata Fritsch and Rich & 1.25 & - & - & - & - & - & - & - & - & - & 10 \\
\hline Rivularia sp. & - & - & - & - & - & - & 0.51 & 0.67 & - & - & 20 \\
\hline Spirulina major Kutz & 0.54 & - & 1.45 & 2.99 & - & - & - & 0.67 & - & - & 40 \\
\hline Synechocystis sp. & 0.36 & - & - & - & - & - & 0.51 & 4.00 & 7.65 & 1.65 & 50 \\
\hline Trichodesmium sp. & 0.36 & - & - & - & - & - & - & 0.67 & - & 0.55 & 30 \\
\hline Woronichinia sp. & - & - & 2.9 & 4.48 & 2.38 & 1.12 & - & - & - & 0.55 & 50 \\
\hline \multicolumn{12}{|l|}{ BACILLARIOPHYCEAE } \\
\hline Amphora ovalis Kutz & - & - & - & - & - & - & 0.51 & 2.00 & 2.04 & 0.55 & 40 \\
\hline Aulacosira granulata & 1.25 & 13.33 & 20.29 & 2.99 & 0.79 & 1.69 & 2.03 & 2.00 & 3.57 & 3.30 & 100 \\
\hline Cocconeis sp. & - & - & - & - & - & - & - & - & - & 1.10 & 10 \\
\hline Cyclotella meneghiniana Kutz & - & 1.67 & 1.45 & - & 0.79 & 0.56 & - & 1.33 & 2.55 & 1.10 & 70 \\
\hline Cymbella minuta Hilse & - & 2.50 & - & - & - & - & - & - & - & - & 10 \\
\hline Fragilaria lapponica Grunow & - & - & - & - & - & - & 0.51 & 0.67 & - & - & 20 \\
\hline Gomphoneis herculana (Ehr.) Cleve & - & 2.50 & 1.45 & - & - & - & - & - & - & - & 20 \\
\hline Gomphonema Augir Ehr. & - & - & - & - & - & - & - & 0.67 & - & 0.55 & 20 \\
\hline Gomphonema sp. & - & 2.50 & 1.45 & - & - & - & 0.51 & 1.33 & 0.51 & - & 50 \\
\hline Gyrosigma sp. & - & 2.50 & - & - & - & - & - & - & 0.51 & - & 20 \\
\hline Navicula cuspidata Kuetzing & - & - & - & - & - & - & - & 0.67 & - & 1.65 & 20 \\
\hline Navicula exigua (Greg.) Muller & - & - & - & - & - & - & 0.51 & - & - & - & 10 \\
\hline Navicula forcipata Grev. & - & - & 2.90 & 7.46 & 0.79 & 0.56 & 3.55 & 4.67 & 3.06 & 1.65 & 80 \\
\hline Navicula lapidosa Krasske & - & - & - & - & - & - & - & 2.00 & - & - & 10 \\
\hline Navicula placentula (Erh.) Kuetzing & - & - & - & - & - & - & - & - & 0.51 & 1.10 & 20 \\
\hline Navicula radiosa Kutzing & - & - & - & - & 0.79 & 0.56 & - & 0.67 & 2.04 & 0.55 & 50 \\
\hline Navicula sp. & 0.54 & 4.17 & - & - & - & - & 0.51 & - & - & - & 30 \\
\hline Nitszchia sp. & - & - & 1.45 & - & - & - & 2.03 & 0.67 & 2.55 & - & 40 \\
\hline Pinnularia similis Hustedt & - & - & - & - & - & - & 0.51 & 0.67 & - & 1.10 & 30 \\
\hline Surirella sp. & - & 2.5 & 1.45 & - & 1.59 & - & 0.51 & 0.67 & 0.51 & 0.55 & 70 \\
\hline Synedra sp. & - & - & - & 1.49 & - & - & - & 0.67 & - & - & 20 \\
\hline \multicolumn{12}{|l|}{ DYNOPHYCEAE } \\
\hline Glenodinium sp. & 0.36 & 2.5 & 2.9 & - & 0.79 & - & - & - & 1.02 & - & 50 \\
\hline Peridinium sp. & 0.36 & 1.67 & - & - & - & - & 1.02 & - & - & - & 30 \\
\hline \multicolumn{12}{|l|}{ CHRYSOPHYCEAE } \\
\hline Mallomonas producta Iwanoff & 0.36 & 3.33 & 2.9 & -1.49 & 2.38 & 2.25 & 0.51 & 0.67 & 2.55 & - & 90 \\
\hline Mallomonas striata Asmund & - & - & - & - & - & 1.69 & 0.51 & 0.67 & 0.51 & 3.30 & 50 \\
\hline Pedinella sp. & - & 2.5 & - & - & - & - & - & - & - & - & 10 \\
\hline \multicolumn{12}{|l|}{ EUGLENOPHYCEAE } \\
\hline Euglena sp. & - & - & - & - & - & - & - & - & 1.02 & - & 10 \\
\hline Phacus acuminatus Stokes & - & 1.67 & - & - & - & 4.49 & 2.03 & 3.33 & 10.2 & 26.37 & 60 \\
\hline
\end{tabular}

P.O. (\%) - Percentage of occurrence. 
Table 3. Phytoplankton species and relative abundance distributed in the mid-column water of Cruzeta, RN Reservoir during the annual cycle of 2004-2005.

\begin{tabular}{|c|c|c|c|c|c|c|c|c|c|c|c|}
\hline & \multicolumn{10}{|c|}{ Relative abundance (\%) } & \multirow{2}{*}{$\begin{array}{l}\text { P.O. } \\
(\%)\end{array}$} \\
\hline & Sept. & Oct. & Nov. & Dec. & Jan. & Fev. & Mar. & Apr. & May & June & \\
\hline \multicolumn{12}{|l|}{ CHLOROPHYCEAE } \\
\hline $\begin{array}{l}\text { Asterococcus superbus } \\
\text { (Cienkowski) Scherffel }\end{array}$ & - & - & - & - & - & - & 0.83 & - & - & - & 10 \\
\hline Botryococcus braunii Kuetzing & 0.58 & 2.42 & - & - & 0.46 & 0.47 & 0.41 & - & - & 0.46 & 60 \\
\hline Chlamydocapsa bacillus (Teiling) Fott & - & - & - & - & - & 0.47 & 2.07 & 0.83 & 0.29 & - & 40 \\
\hline Chlamydocapsa planctonica Fott & - & - & - & - & - & 0.94 & - & - & - & 0.46 & 20 \\
\hline Chorella sp. & - & 25.81 & 16.9 & 9.68 & 18.43 & 9.91 & 3.32 & 3.33 & 4.66 & 12.04 & 90 \\
\hline Closterium lunula Müll & - & - & - & - & 0.46 & - & - & - & - & - & 10 \\
\hline Closterium sp. & - & - & - & - & - & 3.30 & - & - & 0.58 & 0.93 & 30 \\
\hline Coelastrum cambricum Archer & 0.58 & 1.61 & - & - & 1.38 & - & 0.41 & 0.42 & 0.29 & 0.93 & 70 \\
\hline Coelastrum microporum Naegeli & 1.17 & 1.61 & 1.41 & 1.61 & 2.76 & - & 0.41 & 0.42 & 0.29 & 0.93 & 90 \\
\hline Coleochaete pulvinata Smith & - & & - & 1.61 & 1.38 & 0.94 & - & - & 0.29 & 0.46 & 50 \\
\hline Cosmarium subcostatum Nordstedt & 1.17 & - & - & - & - & - & - & - & - & - & 10 \\
\hline Crucigenia sp. & - & - & - & - & 1.84 & 1.42 & 3.73 & - & 0.58 & 0.93 & 50 \\
\hline $\begin{array}{l}\text { Cruciginela rectangularis } \\
\text { (Naegeli) Komárek }\end{array}$ & - & 7.26 & 5.63 & 4.84 & 7.83 & 1.42 & 4.15 & 2.50 & 0.87 & 1.85 & 90 \\
\hline Ctenocladus sp. & - & - & - & - & - & - & 0.41 & 0.42 & - & - & 20 \\
\hline Dictyosphaerium sp. & - & 2.42 & 1.41 & 1.61 & 0.92 & 1.42 & 0.83 & 0.42 & - & 0.93 & 80 \\
\hline Eudorina elegans Ehrenberg & - & - & - & 1.61 & - & - & 0.41 & - & - & 0.46 & 30 \\
\hline Eudorina sp. & 2.92 & 3.23 & 5.63 & 8.06 & 1.84 & 5.66 & 7.47 & 1.67 & 1.17 & 0.46 & 100 \\
\hline $\begin{array}{l}\text { Neospongiococcum gelatinosum } \\
\text { Ettl and Gartner }\end{array}$ & 0.58 & 1.61 & - & - & - & - & - & - & - & - & 20 \\
\hline Oedogonium sp. & - & - & - & - & - & - & - & - & - & 5.56 & 10 \\
\hline Oocystis sp. & 2.92 & 2.42 & 8.45 & 6.45 & 0.92 & 4.25 & 2.90 & 4.17 & 1.46 & 4.63 & 100 \\
\hline Pandorina sp. & 1.17 & 1.61 & - & 3.23 & 5.07 & 3.77 & 5.39 & 0.42 & 2.33 & 1.39 & 90 \\
\hline Phacotus sp. & - & - & - & - & 4.61 & 4.72 & 1.24 & 0.83 & 0.87 & 1.85 & 60 \\
\hline Pleodorina sp. & - & - & - & - & - & - & - & 2.08 & 0.58 & 0.93 & 30 \\
\hline Scenedesmus ecornis Chodat & - & - & - & - & - & 0.47 & - & - & - & - & 10 \\
\hline Scenedesmus obliqus (Turpin) Kuetzing & 1.17 & - & - & - & - & - & - & - & - & - & 10 \\
\hline $\begin{array}{l}\text { Scenedesmus quadricauda } \\
\text { (Turpin) Brébisson }\end{array}$ & - & - & 7.04 & 35.48 & 34.1 & 30.66 & 43.98 & 20.83 & 16.03 & 17.59 & 80 \\
\hline Scenedesmus tibiscensis Uherkovich & - & 1.61 & & - & - & 0.94 & 1.24 & 0.42 & 0.58 & 0.93 & 60 \\
\hline Schroederia setigera (Schroder) Lemm & - & - & - & - & - & - & - & - & 0.29 & - & 10 \\
\hline Sphaerocystis schroeteri Chodat & - & 1.61 & - & - & - & - & - & - & - & - & 10 \\
\hline Staurastrum leptocladum Nordstedt & 1.17 & 3.23 & 2.82 & - & - & - & - & - & - & - & 30 \\
\hline Staurastrum sp. & - & - & - & 1.61 & - & - & - & - & - & - & 10 \\
\hline $\begin{array}{l}\text { Staurodesmus triangularis } \\
\text { (Lagerheim) Teiling }\end{array}$ & - & - & - & - & - & - & - & - & 0.29 & 0.46 & 20 \\
\hline Tetradesmus sp. & - & - & 5.63 & - & 0.46 & - & - & 0.83 & - & - & 30 \\
\hline Tetraedron sp. & - & - & - & - & - & - & - & - & 0.29 & - & 10 \\
\hline \multicolumn{12}{|l|}{ CYANOPHYCEAE } \\
\hline $\begin{array}{l}\text { Anabaena circinalis } \\
\text { Robenhorst ex Bornet et Flahault }\end{array}$ & 0.58 & 1.61 & 1.41 & 1.61 & 0.92 & 0.94 & 0.83 & - & - & 0.46 & 80 \\
\hline Anabaena spiroides Klebahn & 0.58 & 1.61 & - & - & - & - & - & - & - & - & 20 \\
\hline Aphanocapsa sp. & - & - & 1.41 & - & 0.92 & 0.94 & 1.24 & 0.42 & 0.29 & - & 60 \\
\hline $\begin{array}{l}\text { Cylindrospermopsis raciborskii } \\
\text { (Wolosz.) Seen. and Subba }\end{array}$ & 67.25 & - & - & - & - & 0.47 & - & - & 0.29 & - & 30 \\
\hline
\end{tabular}


Table 3. Continued...

\begin{tabular}{|c|c|c|c|c|c|c|c|c|c|c|c|}
\hline & \multicolumn{10}{|c|}{ Relative abundance (\%) } & \multirow{2}{*}{$\begin{array}{l}\text { P.O. } \\
(\%)\end{array}$} \\
\hline & Sept. & Oct. & Nov. & Dec. & Jan. & Fev. & Mar. & Apr. & May & June & \\
\hline Eucapsis sp. & - & - & - & - & - & 1.42 & 0.41 & 0.42 & - & 0.46 & 40 \\
\hline Gloeothece sp. & 1.17 & - & - & - & - & - & - & 0.83 & - & - & 20 \\
\hline Lyngbya sp. & 4.09 & - & - & - & - & 1.89 & 0.83 & 0.83 & 0.58 & - & 50 \\
\hline Oscillatoria limosa Ag. & 1.17 & 1.61 & 1.41 & - & - & - & - & - & 0.29 & - & 40 \\
\hline $\begin{array}{l}\text { Planktolyngbya contorta (Smith) } \\
\text { Echdrateva }\end{array}$ & - & 1.61 & - & - & - & - & - & - & - & - & 10 \\
\hline Planktothrix sp. & - & - & 1.41 & - & 0.46 & 1.42 & - & - & - & - & 30 \\
\hline Pseudoanabaena catenata & - & - & - & - & - & - & - & - & 1.25 & - & 10 \\
\hline Raphidiopsis curvata Fritsch and Rich & 4.09 & - & - & - & - & - & - & - & - & - & 10 \\
\hline Rivularia sp. & - & - & - & - & - & - & - & - & - & 0.46 & 10 \\
\hline Synechocystis sp. & - & - & - & - & - & - & 7.05 & 48.75 & 4.37 & 3.7 & 40 \\
\hline Trichodesmium sp. & - & - & - & - & - & - & - & - & 0.29 & - & 10 \\
\hline Woronichinia sp. & - & - & 2.82 & 4.84 & 0.92 & 1.42 & 1.24 & 1.25 & 0.87 & 0.93 & 80 \\
\hline \multicolumn{12}{|l|}{ BACILLARIOPHYCEAE } \\
\hline Amphora sp. & - & - & - & - & - & - & - & 0.42 & - & 0.46 & 20 \\
\hline Amphora ovalis Kutz & - & - & - & 3.23 & - & - & - & - & - & - & 10 \\
\hline Aulacosira granulata & 2.34 & 21.77 & 22.54 & 4.84 & 1.38 & 4.25 & 0.41 & 0.83 & 0.58 & 4.17 & 100 \\
\hline Cocconeis sp. & - & - & - & - & - & - & - & - & 0.29 & - & 10 \\
\hline Cyclotella meneghiniana Kutz & - & 1.61 & 1.41 & - & - & - & - & 0.42 & 0.58 & 0.46 & 50 \\
\hline Diatoma sp. & - & - & - & - & - & 0.47 & 0.83 & - & 0.29 & - & 30 \\
\hline Gomphoneis herculana (Ehr.) Cleve & - & 3.23 & - & - & - & - & - & - & - & - & 10 \\
\hline Gomphonema sp. & - & 3.23 & - & - & - & 0.47 & - & - & 0.29 & - & 30 \\
\hline Gyrosigma sp. & 1.17 & - & - & 3.23 & - & - & - & 0.42 & - & - & 30 \\
\hline Navicula cuspidata Kuetzing & - & - & - & - & - & - & - & - & 0.29 & - & 10 \\
\hline Navicula forcipata Grev. & - & - & - & 1.61 & - & 0.47 & - & - & 0.58 & - & 30 \\
\hline Navicula lapidosa Krasske & - & - & - & - & - & - & 2.07 & - & - & - & 10 \\
\hline Navicula placentula (Erh.) Kuetzing & - & - & - & - & - & - & - & - & 0.29 & - & 10 \\
\hline Navicula radiosa Kutzing & - & - & - & - & - & - & - & - & 0.58 & - & 10 \\
\hline Navicula sp. & 1.17 & 2.42 & - & - & 0.92 & - & - & 0.83 & 1.17 & 0.93 & 60 \\
\hline Nitszchia sp. & - & - & - & - & - & 0.94 & 0.83 & - & 0.29 & - & 30 \\
\hline Pinnularia similis Hustedt & - & - & - & - & - & - & - & - & 0.29 & - & 10 \\
\hline Pinnularia subcapitata Gregory & - & - & - & - & - & - & - & 0.83 & - & - & 10 \\
\hline Raphoneis sp. & - & - & - & - & - & - & - & - & - & 0.46 & 10 \\
\hline Surirella sp. & - & 1.61 & - & - & - & - & - & 0.42 & - & - & 20 \\
\hline Synedra sp. & - & - & 1.41 & - & 0.92 & - & - & - & - & - & 20 \\
\hline \multicolumn{12}{|l|}{ DINOPHYCEAE } \\
\hline Glenodinium sp. & 1.17 & 1.61 & 4.23 & - & 0.92 & - & - & - & - & - & 40 \\
\hline Peridinium sp. & 0.58 & 1.61 & 1.41 & - & - & - & - & - & - & - & 30 \\
\hline \multicolumn{12}{|l|}{ CHRYSOPHYCEAE } \\
\hline Mallomonas producta Iwanoff & 1.17 & - & - & 4.84 & 2.76 & 0.94 & 0.41 & 0.42 & 1.46 & - & 70 \\
\hline Mallomonas striata Asmund & - & - & - & - & 0.92 & 1.42 & 0.41 & 0.42 & 2.04 & 2.31 & 60 \\
\hline \multicolumn{12}{|l|}{ EUGLENOPHYCEAE } \\
\hline Euglena sp. & - & - & 5.63 & - & - & 0.94 & - & - & - & 0.93 & 30 \\
\hline Phacus acuminatus Stokes & - & - & - & - & 4.61 & 10.85 & 4.15 & 2.92 & 50.44 & 30.09 & 60 \\
\hline
\end{tabular}


Table 4. Phytoplankton species and relative abundance distributed in the bottom water of Cruzeta, RN Reservoir during the annual cycle of 2004-2005.

\begin{tabular}{|c|c|c|c|c|c|c|c|c|c|c|c|}
\hline & \multicolumn{10}{|c|}{ Relative abundance (\%) } & \multirow{2}{*}{$\begin{array}{l}\text { P.O. } \\
(\%)\end{array}$} \\
\hline & Sept. & Oct. & Nov. & Dec. & Jan. & Feb. & Mar. & Apr. & May . & June & \\
\hline \multicolumn{12}{|l|}{ CHLOROPHYCEAE } \\
\hline Botryococcus braunii Kuetzing & - & 3.23 & 1.67 & - & 0.79 & 1.72 & 0.58 & - & - & - & 50 \\
\hline Chlamydocapsa bacillus (Teiling) Fott & - & - & - & - & - & - & 2.92 & - & - & 0.72 & 20 \\
\hline Chorella sp. & - & 1.61 & 18.33 & 8.89 & 3.17 & 4.31 & 4.09 & 5.45 & 3.57 & 3.62 & 90 \\
\hline Closterium sp. & - & - & - & - & 2.38 & 1.72 & - & - & - & - & 20 \\
\hline Coelastrum cambricum Archer & - & 1.61 & - & - & 1.59 & 1.72 & 0.58 & 0.61 & - & - & 50 \\
\hline Coelastrum microporum Naegeli & 1.54 & 1.61 & 1.67 & 1.11 & 3.17 & 2.59 & 0.58 & 1.21 & 0.89 & 0.72 & 100 \\
\hline Coleochaete pulvinata Smith & - & - & & - & 3.97 & 0.86 & - & - & 0.89 & 0.72 & 40 \\
\hline Cosmarium subcostatum Nordstedt & 1.54 & - & - & - & - & - & - & - & - & - & 10 \\
\hline Crucigenia sp. & - & - & - & - & - & 4.31 & 1.17 & 0.61 & - & - & 30 \\
\hline $\begin{array}{l}\text { Cruciginela rectangularis } \\
\text { (Naegeli) Komárek }\end{array}$ & - & - & 3.33 & 2.22 & 3.97 & 1.72 & 1.17 & 2.42 & - & - & 60 \\
\hline Ctenocladus sp. & - & - & - & - & - & - & 0.58 & - & - & - & 10 \\
\hline Desmidium bailey (Ralfs) De Bary & 1.54 & - & - & - & - & - & - & - & - & - & 10 \\
\hline Dictyosphaerium sp. & - & 3.23 & 1.67 & - & 3.97 & 0.86 & 0.58 & 0.61 & 0.89 & - & 70 \\
\hline Eudorina elegans Ehrenberg & - & - & - & - & - & - & 0.58 & - & - & - & 10 \\
\hline Eudorina sp. & 1.54 & 12.90 & 8.33 & 7.78 & 4.76 & 4.31 & 3.51 & 2.42 & 2.68 & 1.45 & 100 \\
\hline Oedogonium sp. & - & - & - & - & - & - & - & - & 0.895 & 55.07 & 20 \\
\hline Oocystis sp. & 1.54 & 9.68 & 5.00 & 12.22 & 2.38 & 0.86 & 1.75 & 4.85 & 0.89 & 1.45 & 100 \\
\hline Pandorina sp. & - & 6.45 & 1.67 & 5.56 & 5.56 & 2.59 & 4.09 & 3.03 & 2.68 & 1.45 & 90 \\
\hline Pediastrum sp. & 3.08 & - & 1.67 & - & - & - & - & - & - & 1.45 & 30 \\
\hline Phacotus sp. & - & - & - & - & 1.59 & 1.72 & - & 1.82 & 2.68 & - & 40 \\
\hline Pleodorina sp. & - & 3.23 & - & - & - & - & - & 4.24 & 1.79 & - & 30 \\
\hline $\begin{array}{l}\text { Scenedesmus quadricauda } \\
\text { (Turpin) Brébisson }\end{array}$ & 10.77 & - & 6.67 & 37.78 & 41.27 & 43.10 & 54.39 & 43.033 & 35.71 & 8.7 & 90 \\
\hline Scenedesmus tibiscensis Uherkovich & - & 3.23 & - & 2.22 & - & 1.72 & 0.58 & 0.61 & - & - & 50 \\
\hline Schroederia setigera (Schroder) Lemm & - & - & - & - & - & - & - & - & 0.89 & - & 10 \\
\hline Sphaerocystis schroeteri Chodat & - & 1.61 & - & - & - & - & 0.58 & - & 0.89 & - & 30 \\
\hline Staurastrum leptocladum Nordstedt & 1.54 & - & 1.67 & - & - & 0.86 & - & - & - & - & 30 \\
\hline $\begin{array}{l}\text { Staurastrum striolatum } \\
\text { (Naegeli) Archer }\end{array}$ & - & 1.61 & - & - & - & - & - & - & - & - & 10 \\
\hline $\begin{array}{l}\text { Staurodesmus triangularis } \\
\text { (Lagerheim) Teiling }\end{array}$ & - & - & - & - & - & - & - & - & 0.89 & - & 10 \\
\hline Tetradesmus sp. & - & - & - & 2.22 & - & - & - & - & - & - & 10 \\
\hline \multicolumn{12}{|l|}{ CYANOPHYCEAE } \\
\hline $\begin{array}{l}\text { Anabaena circinalis } \\
\text { Robenhorst ex Bornet et Flahault }\end{array}$ & - & 1.61 & - & 1.11 & 0.79 & - & - & - & 2.68 & 0.72 & 50 \\
\hline Aphanocapsa sp. & - & - & - & - & 0.79 & - & 1.75 & - & 1.79 & - & 30 \\
\hline $\begin{array}{l}\text { Cylindrospermopsis raciborskii } \\
\text { (Wolosz.) Seen. and Subba }\end{array}$ & 4.62 & - & - & - & - & - & - & - & - & - & 10 \\
\hline Eucapsis sp. & - & - & - & - & 2.38 & - & - & - & 0.89 & - & 20 \\
\hline Lyngbya sp. & 6.15 & - & - & - & - & 1.72 & 0.58 & 0.81 & 1.79 & 2.17 & 60 \\
\hline Oscillatoria limosa Ag. & 1.54 & 1.61 & 1.67 & - & - & - & - & - & - & - & 30 \\
\hline $\begin{array}{l}\text { Planktolyngbya contorta } \\
\text { (Smith) Echdrateva }\end{array}$ & - & 1.61 & 1.67 & - & - & - & - & - & - & - & 20 \\
\hline Planktothrix sp. & - & - & 1.67 & - & - & 0.86 & - & - & - & - & 20 \\
\hline
\end{tabular}


Table 4. Continued...

\begin{tabular}{|c|c|c|c|c|c|c|c|c|c|c|c|}
\hline & \multicolumn{10}{|c|}{ Relative abundance (\%) } & \multirow{2}{*}{$\begin{array}{l}\text { P.O } \\
(\%)\end{array}$} \\
\hline & Sept. & Oct. & Nov. & Dec. & Jan. & Feb. & Mar. & Apr. & May & June & \\
\hline Pseudoanabaena catenata & - & - & - & - & - & 0.86 & 0.58 & 1.21 & - & - & 30 \\
\hline Rivularia sp. & - & - & - & - & - & - & 0.58 & - & - & - & 10 \\
\hline Synechocystis sp. & - & - & - & - & - & 2.59 & 3.51 & 4.24 & - & - & 30 \\
\hline Trichodesmium sp. & 1.54 & - & - & - & - & - & - & - & - & - & 10 \\
\hline Woronichinia sp. & - & - & 3.33 & 3.33 & 1.59 & - & - & - & 1.79 & 1.45 & 50 \\
\hline \multicolumn{12}{|l|}{ BACILLARIOPHYCEAE } \\
\hline Amphora sp. & - & - & - & - & - & - & - & 0.61 & - & - & 10 \\
\hline Amphora ovalis Kutz & 3.08 & - & - & 1.11 & - & - & - & - & - & - & 10 \\
\hline Aulacosira granulata & 41.54 & 32.26 & 20.00 & 1.11 & 7.94 & 6.03 & 2.34 & 7.27 & 4.46 & 7.25 & 100 \\
\hline Ceratoneis sp. & - & 1.61 & - & - & - & - & - & - & - & - & 10 \\
\hline Cyclotella meneghiniana Kutz & 1.54 & 3.23 & 5.00 & 1.11 & - & 0.86 & - & - & 1.79 & - & 60 \\
\hline Epithemia sp. & 1.54 & - & - & - & - & - & - & - & - & - & 10 \\
\hline Fragilaria lapponica Grunow & 1.54 & - & - & - & - & 1.72 & 0.58 & 0.61 & 0.89 & - & 50 \\
\hline Gomphoneis herculana (Ehr.) Cleve & - & - & 1.67 & - & - & - & - & - & - & - & 10 \\
\hline Gomphonema Augir Ehr. & - & - & - & - & - & - & - & - & 0.89 & - & 10 \\
\hline \multicolumn{12}{|l|}{ BACILLARIOPHYCEAE } \\
\hline Gomphonema sp. & - & - & - & - & 0.79 & - & - & 1.21 & - & 1.45 & 30 \\
\hline Gyrosigma sp. & 1.54 & 3.23 & - & - & - & 1.72 & - & - & - & 1.45 & 40 \\
\hline Navicula cuspidata Kuetzing & - & - & - & - & - & - & 0.58 & - & 0.89 & - & 20 \\
\hline Navicula forcipata Grev. & - & - & 1.67 & 3.33 & - & - & - & - & - & - & 20 \\
\hline Navicula lapidosa Krasske & - & - & - & - & - & - & - & 1.82 & 1.79 & - & 20 \\
\hline Navicula radiosa Kutzing & - & - & - & - & - & - & - & 0.61 & 2.68 & 0.72 & 30 \\
\hline Navicula sp. & 1.54 & - & - & - & - & - & 4.09 & 4.24 & 3.57 & 0.72 & 50 \\
\hline Nitszchia sp. & - & - & - & - & - & - & 2.34 & 3.03 & 2.68 & 2.17 & 40 \\
\hline Pinnularia similis Hustedt & - & - & - & - & - & - & 0.58 & 0.61 & - & - & 20 \\
\hline Pinnularia subcapitata Gregory & 1.54 & - & - & 1.11 & - & - & - & - & - & - & 20 \\
\hline Raphoneis sp. & 1.54 & - & - & - & - & - & - & - & - & - & 10 \\
\hline Stauroneis anceps Ehr. & 1.54 & - & - & - & - & - & - & - & - & - & 10 \\
\hline Surirella sp. & 1.54 & - & 1.67 & - & - & - & - & - & - & 1.45 & 30 \\
\hline Synedra sp. & - & - & - & - & 1.59 & 1.72 & - & - & 3.57 & 1.45 & 40 \\
\hline \multicolumn{12}{|l|}{ DYNOPHYCEAE } \\
\hline Glenodinium sp. & 1.54 & 1.61 & 5.00 & - & 1.59 & - & - & - & - & - & 40 \\
\hline Peridinium sp. & - & 1.61 & - & - & - & - & - & - & - & - & 10 \\
\hline \multicolumn{12}{|l|}{ CHRYSOPHYCEAE } \\
\hline Mallomonas producta Iwanoff & 3.08 & 1.61 & 1.67 & 7.78 & 1.59 & - & 0.58 & 0.61 & 1.79 & - & 80 \\
\hline Mallomonas striata Asmund & - & - & - & - & - & - & 0.58 & 0.61 & - & 1.45 & 30 \\
\hline Pedinella sp. & - & - & 3.33 & - & - & - & - & - & - & - & 10 \\
\hline \multicolumn{12}{|l|}{ EUGLENOPHYCEAE } \\
\hline Phacus acuminatus Stokes & - & - & - & - & 2.38 & 6.9 & 1.75 & 1.82 & 9.82 & 2.17 & 60 \\
\hline
\end{tabular}

P.O. (\%) - Percentage of occurrence.

80 taxons were identified at the surface, 77 at mid-depth $(2 \mathrm{~m})$ and 72 at the bottom. Regarding the algal class, Chlorophyceae exhibited the highest number of species at the surface and at mid-depth (34 species) during the rainy season ( 29 and 28 species, respectively). There was a greater number of Bacillariophyceae at the bottom of the reservoir (23) than at the surface and mid-depth (21). These relative abundances were expressed as class wise in three vertical profiles indicating explicitly the succession sequence (Figures 6, 7 and 8).

Tables 2, 3 and 4 present detailed quali-quantative data on species, their relative abundance each month, 

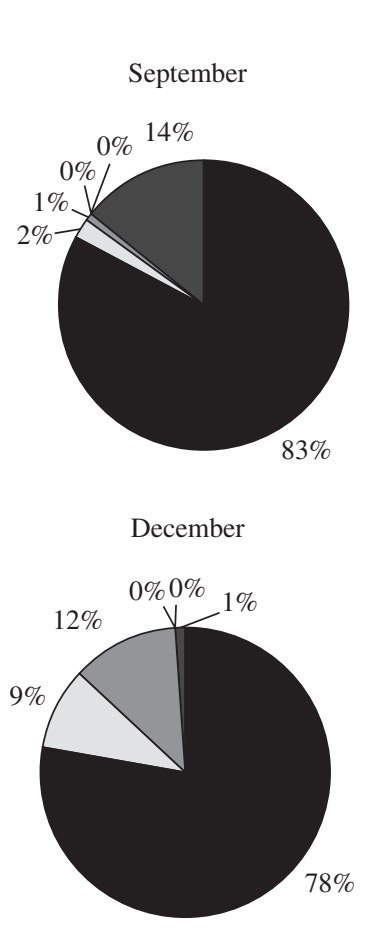

March
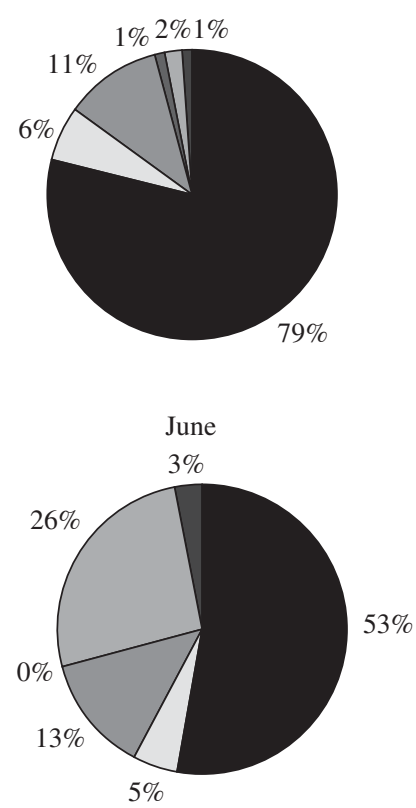

\section{Surface}

October

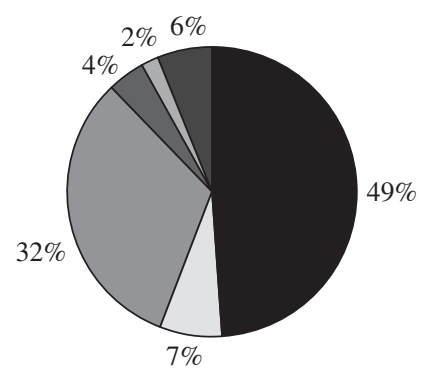

January

$0 \%$

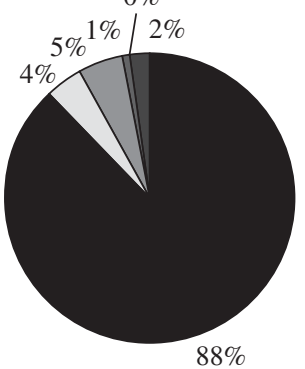

April

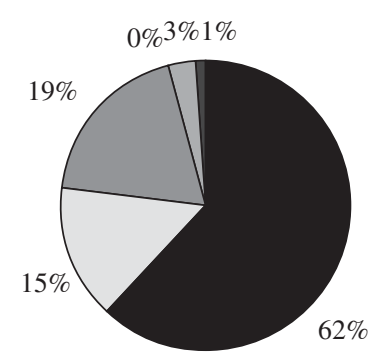

November

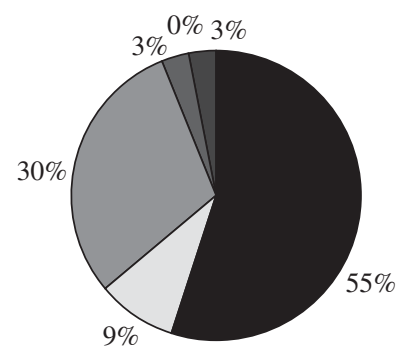

February
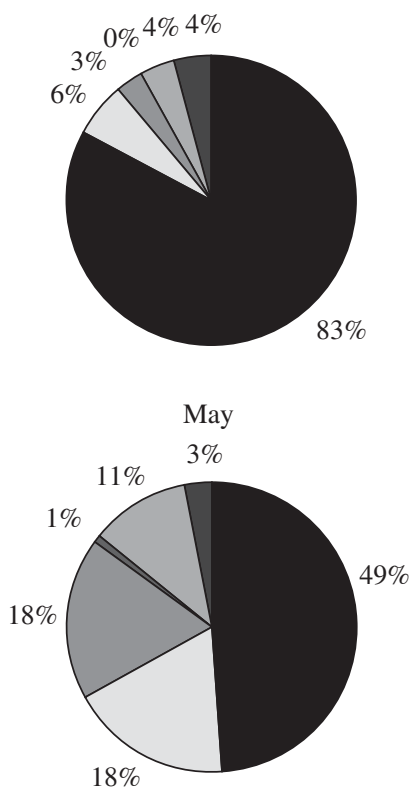

Figure 6. Phytoplankton distribution in surface waters of Cruzeta, RN reservoir from September 2004 to June 2005.

and the frequency of occurrence of phytoplankton in Cruzeta reservoir in the three vertical profiles during the annual cycle of 2004-2005. The largest amount of taxa was found in the rainy season at the surface (68) and middle (66) of the water column. Chlorophyceae dominated the surface during the entire study period except in September, when the Cyanophyceae dominated with
$79 \%$, the most frequent species being the potentially toxic cyanobacteria Cylindrospermopsis raciborskii with a relative abundance of $68.21 \%$. The dominance shift observed among phytoplankton is interesting and often sequential. Chlorophyceae dominance at the surface was followed immediately by Bacillariophyceae, except in February and May. During this period Chlorophyceae 
Mid-Column

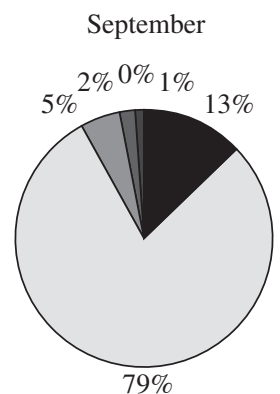

December

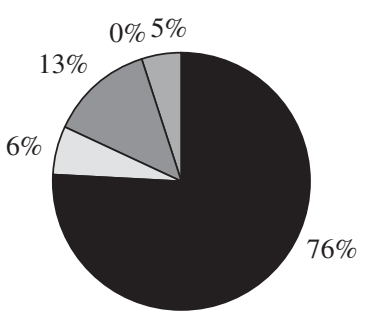

March

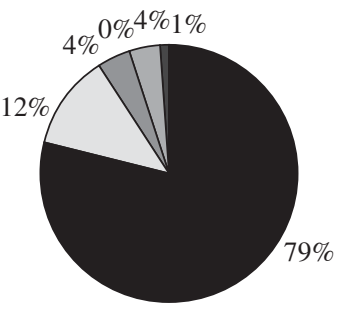

June

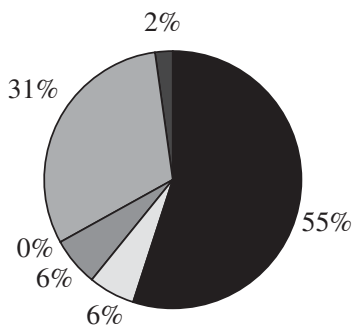

October

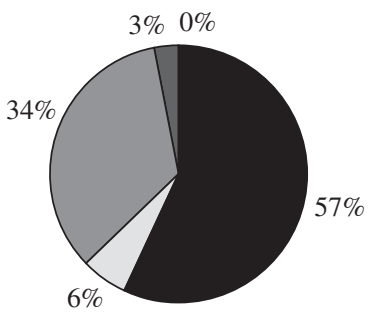

January

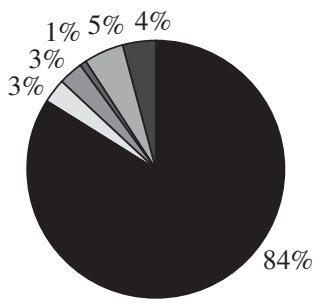

April

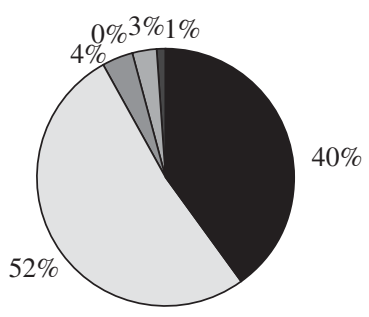

November

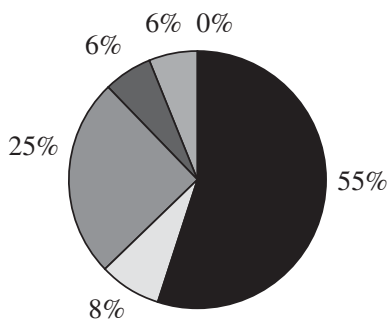

February

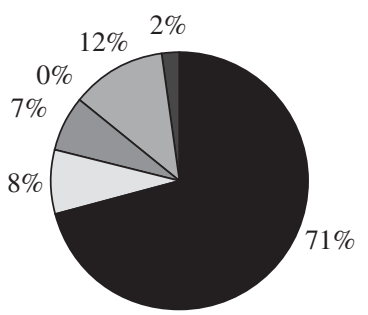

May

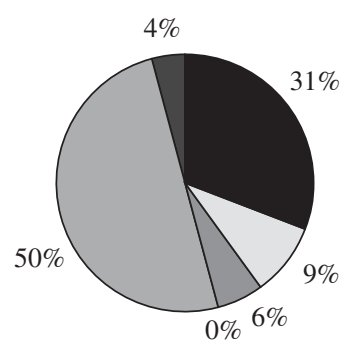

Figure 7. Phytoplankton distribution in mid-column water of Cruzeta reservoir from September 2004 to June 2005.

was followed by Cyanophyceae and in June, when they were followed by Euglenophyceae dominance (26\%). The most abundant Chlorophyceae species at the surface were Scenedesmus quadricauda (Turpin) Brébisson and Chorella sp.. The dominance of phytoplankton groups in the middle of the water column in September, October, November, December and June behaved in the same manner that was observed at the surface. In January and February a dominance of Chlorophyceae was observed, followed by Euglenophyceae, while in March Chlorophyceae (79\%) was dominant, followed by Cyanophyceae (12\%). Cyanophyceae dominated in April (dry period) with 52\%, followed by Chlorophyceae with 31\%. A growth in Euglenophyceae (50\%) occurred in May, when it became dominant, particularly the species Phacus acuminatus Stokes (relative abundance of $50.44 \%$ ), followed by Chlorophyceae with $31 \%$. Chlorophyceae also dominated at the bottom except 
Bottom
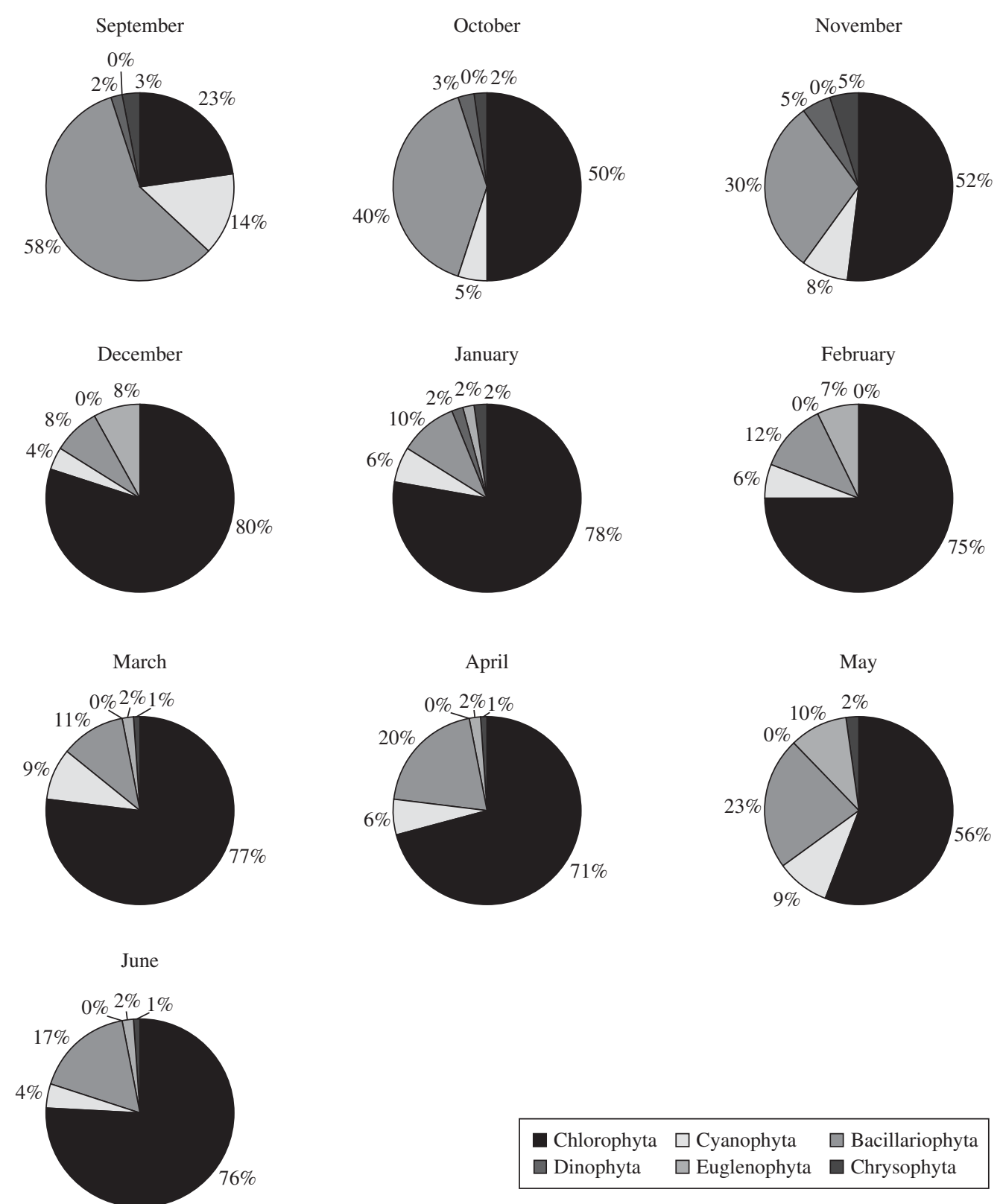

$\begin{array}{ll}\square \text { Chlorophyta } & \square \text { Cyanophyta } \square \text { Bacillariophyta } \\ \square \text { Dinophyta } & \square \text { Euglenophyta } \square \text { Chrysophyta }\end{array}$

Figure 8. Phytoplankton distribution in bottom water of Cruzeta reservoir from September 2004 to June 2005.

in September when there was a strong dominance of Bacillariophyceae. The percentage dominance for these phytoplanktonic species is presented in Table 5 .

Table 6 presents some significant correlation between environmental variables and dominant phytoplankton groups on the spatial-temporal scale. A significant correlation was established for cyanobacterial abundance with water temperature, dissolved oxygen concentration and transparency during the dry period. Bacillariophyceae correlated positively to water transparency and ortho- phosphate levels specifically at the bottom during the dry period and with $\mathrm{pH}$, electrical conductivity and orthophosphate during the wet period. The other dominant group, Chlorophyceae, positively correlated to $\mathrm{pH}$, nitrate and electrical conductivity.

Shannon-Wienner (1949) species diversity and Pielou's (1975) evenness index were determined for the phytoplankton community of the Cruzeta reservoir at the surface, mid-column $(2 \mathrm{~m})$ and bottom, and the indices varied considerably (Table 7). In general, the greatest 
Table 5. Density of species (ind. $\mathrm{mL}^{-1}$ ) dominance at surface, mid-column and bottom waters of Cruzeta, $\mathrm{RN}$ reservoir during September/04 to June/05.

\begin{tabular}{|c|c|c|c|c|c|c|c|c|c|c|c|}
\hline & \multicolumn{10}{|c|}{ Surface } & \multirow{2}{*}{$\begin{array}{l}\text { P.O } \\
(\%)\end{array}$} \\
\hline & Sept. & Oct. & Nov. & Dec. & Jan. & Feb. & Mar. & Apr. & May & June & \\
\hline \multicolumn{12}{|l|}{ CHLOROPHYCEAE } \\
\hline Chlorella sp. & - & 1.100 & 700 & 1.600 & 1.400 & 1.000 & 700 & 400 & 1.5002 & 2.700 & 90 \\
\hline Coelastrum cambricum Archer & 100 & 100 & - & - & 100 & 200 & 100 & 100 & 100 & 100 & 80 \\
\hline Coelastrum microporum Naegeli & 350 & 150 & 100 & 200 & 600 & 200 & 200 & 100 & 300 & 200 & 100 \\
\hline $\begin{array}{l}\text { Cruciginela rectangularis } \\
\text { (Naegeli) Komárek }\end{array}$ & 150 & & -800 & 300 & 1.900 & 400 & 700 & 500 & 500 & 300 & 90 \\
\hline Dictyosphaerium sp. & 950 & 200 & 100 & 200 & 100 & 600 & 700 & 300 & 100 & 100 & 100 \\
\hline Eudorina sp. & 250 & 150 & 400 & 900 & 1.100 & 700 & 600 & 200 & 400 & 400 & 100 \\
\hline Oocystis sp. & 750 & 350 & 600 & 400 & 500 & 700 & 1.00 & 800 & 300 & 500 & 100 \\
\hline Pandorina sp. & 300 & 100 & 400 & 300 & 500 & 2.200 & 500 & 100 & 100 & 400 & 100 \\
\hline $\begin{array}{l}\text { Scenedesmus quadricauda } \\
\text { (Turpin) Brébisson }\end{array}$ & - & & -300 & 1.300 & 4.100 & 5.800 & 9.300 & 4.300 & 4.9003 & 3.300 & 80 \\
\hline \multicolumn{12}{|l|}{ CYANOPHYCEAE } \\
\hline $\begin{array}{l}\text { Anabaena circinalis Robenhorst ex } \\
\text { Bornet et Flahault }\end{array}$ & 100 & 100 & - & 100 & 100 & 500 & 100 & 200 & 200 & 100 & 90 \\
\hline \multicolumn{12}{|l|}{ BACILLARIOPHYCEAE } \\
\hline Aulacoseira granulata & 350 & 800 & 1.400 & 200 & 100 & 300 & 400 & 300 & 700 & 600 & 100 \\
\hline Navicula forcipata Grev. & - & & -200 & 500 & 100 & 100 & 700 & 700 & 600 & 300 & 80 \\
\hline \multicolumn{12}{|l|}{ CHRYSOPHYCEAE } \\
\hline \multirow[t]{2}{*}{ Mallomonas producta Iwanoff } & 100 & 200 & 200 & 100 & 300 & 400 & 100 & 100 & 500 & - & 90 \\
\hline & \multicolumn{11}{|c|}{ Mid-Collum } \\
\hline \multicolumn{12}{|l|}{ CHLOROPHYCEAE } \\
\hline Chorella sp. & - & 1.600 & 1.200 & 600 & 4.000 & 2.100 & 800 & 800 & 1.6002 & 2.600 & 90 \\
\hline Coelastrum microporum Naegeli & 200 & 100 & 100 & 100 & 600 & - & 100 & 100 & 100 & 200 & 90 \\
\hline $\begin{array}{l}\text { Cruciginela rectangularis } \\
\text { (Naegeli) Komárek }\end{array}$ & - & 450 & 400 & 300 & 1.700 & 300 & 1.000 & 600 & 300 & 400 & 90 \\
\hline Dictyosphaerium sp. & - & 150 & 100 & 100 & 200 & 300 & 200 & 100 & - & 200 & 80 \\
\hline Eudorina sp. & 500 & 200 & 400 & 500 & 400 & 1.200 & 1.800 & 400 & 400 & 100 & 100 \\
\hline Oocystis sp. & 500 & 150 & 600 & 400 & 200 & 900 & 700 & 1.000 & 500 & 1.000 & 100 \\
\hline Pandorina sp. & 200 & 100 & - & 200 & 1.100 & 800 & 1.300 & 100 & 800 & 300 & 90 \\
\hline $\begin{array}{l}\text { Scenedesmus quadricauda } \\
\text { (Turpin) Brébisson }\end{array}$ & - & - & 500 & 2.200 & 7.400 & 6.500 & 10.600 & 5.000 & 5.5003 & 3.800 & 80 \\
\hline \multicolumn{12}{|l|}{ CYANOPHYCEAE } \\
\hline $\begin{array}{l}\text { Anabaena circinalis Robenhorst ex } \\
\text { Bornet et Flahault }\end{array}$ & 100 & 100 & 100 & 100 & 200 & 200 & 200 & - & - & 100 & 80 \\
\hline Woronichinia sp. & - & - & 200 & 300 & 200 & 300 & 300 & 300 & 300 & 200 & 80 \\
\hline \multicolumn{12}{|l|}{ BACILLARIOPHYCEAE } \\
\hline \multirow[t]{2}{*}{ Aulacoseira granulata } & 400 & 1.350 & 1.600 & 300 & 300 & 900 & 100 & 200 & 200 & 900 & 100 \\
\hline & \multicolumn{11}{|c|}{ Bottom } \\
\hline \multicolumn{12}{|l|}{ CHLOROPHYCEAE } \\
\hline Chorella sp. & - & 100 & 1.100 & 800 & 400 & 500 & 700 & 900 & 400 & 500 & 90 \\
\hline Coelastrum microporum Naegeli & 100 & 100 & 100 & 100 & 400 & 300 & 100 & 200 & 100 & 100 & 100 \\
\hline Eudorina sp. & 100 & 800 & 500 & 700 & 600 & 500 & 600 & 400 & 300 & 200 & 100 \\
\hline Oocystis sp. & 100 & 600 & 300 & 1.100 & 300 & 100 & 300 & 800 & 100 & 200 & 100 \\
\hline Pandorina sp. & - & 400 & 100 & 500 & 700 & 300 & 700 & 500 & 300 & 200 & 90 \\
\hline $\begin{array}{l}\text { Scenedesmus quadricauda (Turpin) } \\
\text { Brébisson }\end{array}$ & 200 & - & 400 & 3.400 & 5.200 & 5.000 & 9.300 & 7.100 & 4.000 & 1.200 & 90 \\
\hline
\end{tabular}


Table 5. Continued...

\begin{tabular}{|c|c|c|c|c|c|c|c|c|c|c|c|}
\hline & \multicolumn{10}{|c|}{ Bottom } & \multirow{2}{*}{$\begin{array}{l}\text { P.O. } \\
(\%)\end{array}$} \\
\hline & Sept. & Oct. & Nov. & Dec. & Jan. & Feb. & Mar. & Apr. & May & June & \\
\hline \multicolumn{12}{|l|}{ BACILLARIOPHYCEAE } \\
\hline Aulacosira granulata & 2.700 & 2.000 & 1.200 & 100 & 1.000 & 700 & 400 & 1.200 & 500 & 1.000 & 100 \\
\hline \multicolumn{12}{|l|}{ CHRYSOPHYCEAE } \\
\hline Mallomonas producta Iwanoff & 200 & 100 & 100 & 700 & 200 & - & 100 & 100 & 200 & - & 80 \\
\hline \multicolumn{12}{|l|}{$\begin{array}{l}\text { Criteria of dominance based on } \\
\text { Matucci and Colma (1982): }\end{array}$} \\
\hline $76-100 \%$ Dominants & & & & & & & & & & & \\
\hline
\end{tabular}

Table 6. Pearson correlation coefficient values between abiotic factors and phytoplankton classes $(\mathrm{P}<0.01)$.

\begin{tabular}{|c|c|c|c|c|c|c|}
\hline \multirow[t]{2}{*}{ Variables } & \multicolumn{3}{|c|}{ Dry Season } & \multicolumn{3}{|c|}{ Rainy Season } \\
\hline & Surface & Mid-Column & Bottom & Surface & Mid-Column & Bottom \\
\hline Temperature and Bacillariophyceae & -1.00 & -0.82 & - & - & - & - \\
\hline Cyanophyceae and Nitrate & -0.96 & - & - & - & - & - \\
\hline Euglenophyceae and Orthophosphate & 0.98 & - & - & - & - & - \\
\hline Chlorophyceae and Dissolved Oxygen & 0.80 & -0.90 & - & - & - & - \\
\hline Euglenophyceae and $\mathrm{pH}$ & - & - & - & 0.98 & - & - \\
\hline $\begin{array}{l}\text { Orthophosphate and } \\
\text { Bacillariophyiceae / Euglenophyceae }\end{array}$ & - & - & - & $0.87 / 0.85$ & - & - \\
\hline Chlorophyceae and Transparency & - & 0.99 & -0.97 & - & - & - \\
\hline Bacillariophyceae and Rainfall & - & -0.98 & - & - & - & - \\
\hline $\begin{array}{l}\text { Cyanophyceae and } \\
\text { Transparency / Conductivity }\end{array}$ & - & $0.86 /-0.82$ & - & - & - & - \\
\hline $\begin{array}{l}\text { Cyanophyceae and } \\
\text { Conductivity / Orthophosphate }\end{array}$ & - & - & - & - & $0.82 / 0.83$ & - \\
\hline $\begin{array}{l}\text { Chlorophyceae and } \\
\text { Conductivity / Orthophosphate }\end{array}$ & - & - & - & - & $0.83 /-0.87$ & - \\
\hline Chlorophyceae and $\mathrm{pH} /$ Conductivity & - & - & $0.93 / 0.97$ & - & - & - \\
\hline Cyanophyceae and Ammonium & - & - & 0.97 & - & - & - \\
\hline Bacillarioficeae and Transparency & - & - & 0.97 & - & - & - \\
\hline Bacillariophyceae and $\mathrm{pH}$ & - & - & -0.99 & - & - & 0.83 \\
\hline Bacillariophiceae and Orthophosphate & - & - & 0.92 & - & - & 0.93 \\
\hline Bacillariophyceae and Conductivity & - & - & -0.99 & - & - & 0.98 \\
\hline Bacillariophyceae and Nitrate & - & - & -0.8 & - & - & - \\
\hline
\end{tabular}

Table 7. Species richness, Species diversity, Dominance, Equitability and Total Density of species during the annual cycle of 2004-2005.

\begin{tabular}{|c|c|c|c|c|c|c|c|c|c|c|c|c|c|c|c|}
\hline \multirow[t]{2}{*}{ Months } & \multicolumn{3}{|c|}{ Richness } & \multicolumn{3}{|c|}{ Diversity } & \multicolumn{3}{|c|}{ Dominance } & \multicolumn{3}{|c|}{ Equitability } & \multicolumn{3}{|c|}{ Total density (ind.mL ${ }^{-1}$ ) } \\
\hline & $\mathbf{S}$ & M & B & $\mathbf{S}$ & M & B & $\mathbf{S}$ & M & B & $\mathbf{S}$ & M & B & $\mathbf{S}$ & M & B \\
\hline Sept./04 & 6.75 & 5.20 & 6.29 & 2.22 & 2.29 & 3.43 & 0.68 & 0.67 & 0.42 & 1.49 & 1.68 & 2.45 & 28.000 & 17.100 & 6.500 \\
\hline Oct./04 & 7.68 & 6.33 & 5.54 & 4.44 & 3.75 & 3.99 & 0.18 & 0.26 & 0.32 & 3.01 & 2.68 & 2.97 & 6.000 & 6.200 & 6.200 \\
\hline Nov./04 & 6.51 & 4.93 & 5.82 & 4.02 & 3.68 & 3.60 & 0.20 & 0.23 & 0.20 & 2.84 & 2.83 & 2.64 & 6.900 & .100 & 6.000 \\
\hline Dec./04 & 3.66 & 4.48 & 4.05 & 3.35 & 3.39 & 3.13 & 0.24 & 0.35 & 0.38 & 2.85 & 2.70 & 2.54 & 6.700 & 6.200 & 9.000 \\
\hline Jan./05 & 6.10 & 6.00 & 5.37 & 3.45 & 3.46 & 3.45 & 0.33 & 0.34 & 0.41 & 2.44 & 2.42 & 2.53 & 12.300 & 21.700 & 12.600 \\
\hline Feb./05 & 5.88 & 7.17 & 6.15 & 3.53 & 3.57 & 3.46 & 0.33 & 0.31 & 0.43 & 2.49 & 2.37 & 2.45 & 17.800 & 21.200 & 11.600 \\
\hline Mar./05 & 10.01 & 6.62 & 7.09 & 3.58 & 3.08 & 3.06 & 0.47 & 0.44 & 0.54 & 2.18 & 2.09 & 2.05 & 19.700 & 24.100 & 17.100 \\
\hline Apr./05 & 10.30 & 6.85 & 6.40 & 4.50 & 2.75 & 3.41 & 0.29 & 0.49 & 0.43 & 2.74 & 1.84 & 2.36 & 15.000 & 24.000 & 16.500 \\
\hline May/05 & 9.55 & 8.82 & 7.41 & 4.30 & 3.13 & 3.89 & 0.25 & 0.50 & 0.36 & 2.65 & 1.94 & 2.61 & 19.600 & 34.300 & 11.200 \\
\hline June/05 & 3.35 & 3.63 & 2.75 & 3.35 & 3.63 & 2.75 & 0.26 & 0.52 & 0.55 & 2.15 & 2.39 & 0.66 & 18.200 & 21.600 & 13.800 \\
\hline
\end{tabular}

S - Surface; M - Mid-column; B - Bottom; P.O. (\%) - Percentage of occurrence. 
values of specific diversity, and equitability (similarity), richness and dominance were observed at the surface and bottom waters than at mid-column. In relation to the dry and wet seasons, there was greater diversity and less evenness during the rainy period. In September 2004, an invasive species of cyanobacterium, Cylindrospermopsis raciborskii, dominated both at the surface and the middle of the water column and never repeated. An elevated dominance of Scenedesmus quadricauda, Oocystis sp. and Oedogonium sp., members of Chlorophyceae was also observed at the bottom in March and June of 2005. The dominance of these three species determined a reduction in the diversity values in the dry/wet phases of the annual cycle. Phytoplankton species diversity is addressed in relation to three hydroperiods with six replicates for each period which reflect the level of organization of the phytoplankton community of Cruzeta, RN reservoir. Phytoplankton species diversity and evenness reflect important processes such as growth, sedimentation, possible grazing losses and nutrient assimilation, which varied markedly in relation to spatial heterogeneity and the dry/wet annual cycle. The present study reveals three hydroperiods, such as the dry period (Sept. to Dec. 2004), the wet period (Jan. to Feb. 2005 and Apr. to June 2005) and the peak rainy period of March 2005. The species diversity and equitability indices were greatly reduced in March 2005 largely due to low attenuation of light due to the accumulation of high suspended materials near the surface.

\section{Discussion}

Shallow water reservoirs (mean depth $<6 \mathrm{~m}$ ) are dominant freshwater systems in the Rio Grande do Norte State and are more vulnerable to human impact on phytoplankton dynamics and inland fisheries of the State (Chellappa and Chellappa, 2004). The spatio-temporal distributions of major phytoplankton taxa were quantified to estimate the relative contribution to abundance, cyanobacterial bloom dynamics and chlorophyll biomass in the oligo-mesotrophic Cruzeta, $\mathrm{RN}$ reservoir during the annual cycle of 2004-2005. The results are discussed within the framework of shallow water freshwater ecosystems principle and to highlight how phytoplankton community structure, diversity and biomass respond to clear and turbid water conditions during the dry and wet cycles of semiarid climate (Bouvy et al., 2003).

Depth influences sinking loss of phytoplankton and its suspension to surface layer in deep water reservoirs and lakes, while the pronounced effect experienced by phytoplankton under light regime in shallow waters is because of the light attenuation coefficient of the mixed layer (Shaffer, 2004). The present study indicates that the mean depth was never more than $4 \mathrm{~m}$ throughout the study period, of mostly an isothermal characteristic with no marked stratification. The light regime experienced by the phytoplankton varied on a seasonal basis, with more light penetration in the dry than wet period and the chlorophyll biomass linearly linked to the light regime than to phytoplankton abundance. Such findings are similar to the results of the Parelhas, $\mathrm{RN}$ reservoir (Chellappa et al., 2006). The polymictic nature of semiarid reservoirs where elevated daytime and low nighttime temperatures characterize the diurnal cycle, favor important convective movements in the mixing of the water column and the consequent rise of nutrients to the water surface (Bouvy et al., 1999; Chellappa and Costa, 2003). The earlier study of Barbosa and Tundisi (1980) registered such phenomena in other shallow water lakes in Brazil and they considered it as an efficient mechanism of the recycling process in tropical waters and consequently improved primary production. The results of nutrient values found in the present study is akin to the research performed by the earlier studies of Chellappa and Chellappa (2004) for the Rio Grande do Norte State, in which the data on surface and bottom concentrations of phosphorous were generally similar, with the exception of September, when phosphorous concentration at the bottom was much greater than that at the surface.

Cruzeta reservoir of Rio Grande do Norte maintained a high phytoplankton species richness (90 taxons) during the 2004-2005 annual cycle, which is very nearly similar to the report of Train et al. (2005) on algal species in three reservoirs in the Parana basin-Irai, Salto do Vau and Rosana, where phytoplankton was distributed in the following classes: Chlorophyceae (32), Cyanophyceae (17), Bacillariophyceae (15), Chrysophyceae (5), Zygnematophyceae (5), Xanthophyceae (3) and Dinophyceae (2). However, Huszar and Giani (2004) pointed out that the phytoplankton community might present a different pattern in vertical distribution, as a result of the physical structuring of the water mass and the capacity of algae to regulate their position through specific adaptive strategies. The present study demonstrates that Bacillariophyceae was more abundant in mid-column and bottom waters, while Chlorophyceae and Cyanobacteria are more abundant in the surface and mid-column of the reservoir mostly as a response to surface/volume ratio of these groups and the differential buoyancy mechanism.

Bicudo et al. (1999) attributed the high diversity of species found to the mixing of the water column, which changes the progress of succession to a condition of equilibrium. Thus, the presence of an unbalanced community triggers high diversity indices. Moran et al. (1997) observed that the biodiversity indicator is fundamental in assessing ecological stability in environments. The high diversity values obtained in the present study can be considered an indicator of the stability of Cruzeta, RN reservoirs because of two reasons: it maintained multispecies phytoplankton in the annual cycle, as evidenced by moderate indices of equitability and the restriction of high dominance index by cyanobacteria only on a rare occasion (September, 2004).

The role of rainfall has been emphasized as a fundamental factor in the ecologic processes of tropical 
ecosystems. Through the contribution of nutrients and materials in suspension (runoff), light penetration is reduced and chemical conditions and mixing of the water, triggers the succession process. Giani and Figueiredo (1999), in a study performed at the Pampulha reservoir, Minas Gerias, Brazil, reported that rainfall acts as a diluting factor and, at the same time, a disturber of aquatic communities, having a strong influence on the composition of phytoplankton species as well as on total biomass.

Nutrient dynamics is usually linked to seasonal variation and the degree of allochthonous input to the reservoirs. Nutrient availability in shallow lakes differed profoundly between shallow polymictic lakes to deep stratified lakes (Scheffer, 2004). The nutrient loss is continuous through sinking in deep waters from epilimnion to hypolimnion, while shallow waters frequently replenish nutrients through efficient recycling and elevated mixing regime (Barbosa and Tundisi, 1980; Scheffer, 2004). The low N/P ratio coupled with high temperature favor cyanobacterial blooms (Smith, 1983), but in the present study, such observation was made only during a single incidence of Cylindrospermopsis raciborskii bloom in September 2004 while the rest of the results tend to contradict the influence of low N/P ratio.

Shapiro (1973) attributed the dominance of Cyanophyceae in fresh water reservoirs mainly to water temperature, light, $\mathrm{CO}_{2}$ and $\mathrm{pH}$. Chellappa (1990) argued that the semi-arid reservoirs of northeast Brazil had suffered greatly from the effects of the environmental impact of cultural eutrophication, which favor the drastic increase in Cyanophyceae populations and cause reduced diversity in microalgae species. Branco and Senna (1996) reported an abundant presence of Cylindrospermopsis raciborskii in Paranoá Lake in Brasilia in the last three decades of the $20^{\text {th }}$ century, reaching densities of $1.0 \times 10^{8}$ trichomes per liter that represented the main cause of high phytoplankton densities. The presence and the abundance of C. raciborskii were also reported for a eutrophic ecosystem in São Paulo state for short and long-term periods (Tucci and Sant' ana, 2003). According to Padisák (1997), Cylindrospermopsis raciborskii has been considered an expanding species in various parts of the world, and presented potential toxicity and a tendency to bloom formation. Its ecological success is due to various factors: migration capacity in the water column; tolerance to low luminosity; high capacity for phosphate and ammonium absorption; capacity to fix atmospheric nitrogen; herbivory resistance; and the ability of this alga to fix inorganic carbon at high $\mathrm{pH}$ values or the capacity to use bicarbonates directly as a source of carbon (Golterman et al., 1978).

The Cruzeta reservoir exhibits great phytoplankton diversity with a total of 90 taxa distributed into 6 classes. Chlorophyceae dominated most of the time, the most frequent taxons were: Scenedesmus quadricauda, Chlorella sp., Eudorina sp., Pandorina sp., Dictyosphaerium sp. and Oocystis sp. Toxic cyanobac- teria, such as Cylindrospermopsis raciborskii, dominate occasionally and preferentially during the dry period, possibly as a result of increased temperature and transparency. Bacillariophyceae, such as Aulacoseria granulata, is frequent in the environment. The increase in the Phacus acuminatus population (Euglenophyceae) coincided with the increase in $\mathrm{pH}$ and the greater concentrations of orthophosphate. The reservoir is characteristically an oligo-mesotrophic environment.

Acknowledgements - We thank CAPES/MEC for the scholarship awarded to one of us (J.L.M.B), CNPq (Proc. No.475619/2004-8) for the research Grant and UFRN for the overall facilities provided.

\section{References}

APHA. American Public Health Association, 1985. Standard methods for examination of water and wasterwater. 16 ed. $1527 \mathrm{p}$.

AZEVEDO, SMFO., EVANS, W., CARMICHAEL, WW. and NAMIKOSHI, W., 1994. First report of Microcystins from a Brazilian isolate of the Cyanobacterium Microcystis aeruginosa. Jour. Appl. Phycol., vol. 6, no. 3, p. 261-265.

BARBOSA, FAR. and TUNDISI, JG. 1980. Primary production of phytoplankton and environmental characteristics of a shallow quaternary lake at Eastern Brazil. Arch.Hydrobiol., vol. 90, no. 2, p. 139-161.

BARBOSA, FAR., BICUDO, CEM. and HUSZAR, VLM. 1995. Phytoplankton studies in Brazil: Community structure variation and diversity. In: TUNDISI, JG., BICUDO, CEM. and TUNDISI, JG (Eds.). Limnology in Brasil. Rio de Janeiro: Academia Brasileira de Ciências; Sociedade Brasileira de Limnologia. p. 19-36.

BICUDO, CEM., RAMIREZ, JJ. and TUCCI, A. 1999. Dinâmica de populações fitoplanctônica em ambiente eutrofizado: Lago das Garças, São Paulo. In: HENRY (Ed.). Ecologia de Reservatórios. Botucatu/SP: Ed. FUNDIBIO, FAPESP. p. 449-508.

BOUVY, M., FALÇÃO, D., MARINHO, M., PAGANO, M. and MOURA, A. 2000. Occurrence of Cylindrospermopsis (Cyanobacteria) in 39 Brazilian tropical reservoirs during the 1998 drought. Aquat. Microbiol. Ecol., vol. 23, no.1, p. 13-27.

BOUVY, M., NASCIMENTO, SM., MOLICA, RJR., FERREIRA, A., HUSZAR, VL. and AZEVEDO, SMFO. 2003. Limnological features in Tapacurá reservoir (northeast Brazil) during a severe drought. Hydrobiol., vol. 493, no. 1, p. 115-130.

BRANCO, CWC. and SENNA, PAC., 1996. Phytoplankton composition, community structure and seasonal changes in a tropical reservoir (Paranoá Reservoir, Brasil). Algol. Stud., vol. 81 , no.1, p. 69-84.

CARPENTER, SR., KITCHELL, JF. and HODGSON, JR. 1985. Cascading trophic interactions and lake productivity. Bioscien. vol. 35, no.10, p. 634-639.

CHELLAPPA, NT. 1990. Phytoplankton species composition, chlorophyll biomass and primary productivity of Jundiaí reservoir, Northeast., Brazil. before and after eutrophication. Acta Hydrobiol. vol. 32, no. 1, p. 75-91 
CHELLAPPA, NT., AMORIM, JMF., BEZERRA, TA., OLIVEIRA, VC. and COSTA, IA. 1996. Studies on microalgae of Rio Grande do Norte, Brazil. A comparison of phytoplankton assemblages of oligotrophic and eutrophic lake. Beih. Beih. Nova Hedwigia, vol. 12, (Special edition), p. 513-542.

CHELLAPPA, NT., CHELLAPPA, T., LIMA, AKA., BORBA, JLM., SOUZA, PVV. and CHELLAPPA, S. 2006. Ecology of freshwater phytoplankton assemblages from a tropical reservoir of Northeastern Brazil. International Jour. Lakes Res. vol. 1, no. 1 , p. $61-73$

CHELlAPPA, NT. and COSTA, MAM. 2003. Dominant and co-existing species of cyanobacteria from a eutrophicated reservoir of Rio Grande do Norte State. International Journal of Ecology. Acta Oecol. vol. 24, no 1, p. 3-10.

CHELLAPPA, NT, MARINHO, IR. and COSTA, MAM. 2000. Harmful cyanobacterial blooms from semiarid freshwater ecosystems of Northeast Brazil. Aust. Soc. Limnol. Newsletter, vol. 38 , no. 1 , p. $45-49$

CHELLAPPA, S. and CHELLAPPA, NT. 2004. Ecology and Reproductive plasticity of Amazonian Cichlid fishes introduced to the freshwater ecosystems of the semiarid Northeast Brazil. In: Kaul, BR. (Ed.). Advances in Fish and Wildlife Ecology and Biology. New Delhi, India: Daya Publications. p. 45-57.

CODY, ML. 1996. Introduction to long-term community ecological studies. In: CODY, ML. and SMALLWOOD, JA. (Eds.). Long-term studies of vertebrate communities. San Diego, USA: Academic Press. p. 1-15.

GIANI, A. and FIGUEIREDO, CC. 1999. Recorrência de padrões sazonais do fitoplâncton num reservatório eutrófico: reservatório da Pampulha (MG). In HENRY, R. (Ed.). Ecologia de Reservatórios. Botucatu/SP: Ed. FUNDIBIO, FAPESP. p. $531-550$

GOLTERMAN, HL., CLYMO, RS. and OHNSTAD, MAM., 1978. Methods for physical and chemical analysis of Freshwaters. IBP Handbook. Oxford: Blackwell Sci. Publ. $215 \mathrm{p}$.

HENRY, R. 1999. Ecologia de Açudes: Estrutura, função e Aspectos Sociais. Botucatu, SP: Ed. FUNDIBIO; FAPESP. 799 p.

HUSZAR, VLM., SILVA, LHS., MARINHO, M., DOMINGOS, P. and SANTANNA, CL. 2000. Cyanoprokaryote assemblages in eight productive tropical Brazilian waters. Hydrobiol. vol. 424, no. 1-3, p. 67-77.

HUSZAR, VLM. and GIANI, A. 2004. Amostragem fitoplanctônica em águas continentais: Reconhecimento de padrões espaciais e temporais. In BICUDO, CEM. and BICUDO, DC. (Orgs.). Amostragem em Limnologia. São Carlos: Ed. Rima. p. 133-147, cap. 8

KIMMEL, BL., LIND, OT. and PAULSON, LJ. 1999. Reservoir primary production. In THORNTON, KW., KIMMEL, BL., PAYNE, FE. (Eds.). Reservoir limnology: ecological perspectives. New York: J. Wiley \& Sons. p. 133-194.

MEDEIROS, JL. 2005. Caracterização da Comunidade Fitoplanctônica do Açude de Cruzeta/RN. Pós-graduação em
Bioecologia aquática. Natal: Universidade Federal do Rio Grande do Norte. 141 p. [Dissertação Mestrado].

MEIJER, ML., VAN NES, EH., LAMMENS, EHHR. and GULATI, R. 1994. The consequences of a drastic fish stock reduction in small shallow lake Wolderwijd, The Netherlands - Can we understand what happened?. Hydrobiol.., vol. 276, no. 1, p. $31-42$

MORAN, D., PEARCE, D. and WENDELAAR, S. 1997. Investing in biodiversity: an economic prospect on global priority. Biodivers. Conserv. vol. 6, no. 9, p. 1219-1243.

NOGUEIRA, MG., JORCIN, A., VIANNA, NC. and BRITTO, YCT. 2005. Reservatórios em cascata e os efeitos na limnologia e organização das comunidades Bióticas (Fitoplâncton, Zooplâncton e Zoobentos) - Um estudo de caso no Rio Paranapanema (SP/PR). In NOGUEIRA, MG., HENRY, R. and JORCIN, A. (Orgs.). Ecologia de reservatórios. São Carlos, SP: RiMa. p. 83-125.

PADISÁK, J. 1997. Cylindrospermopsis raciborskii (Woloszynnska) Seenayya et Subba Raju, an expanding, highly adaptive cyanobacterium: worldwide distribution and review of its ecology. Arch. Für. Hybrobiology, vol. 107, no. 4, p. 563-593.

PINTO-COELHO, RM., AZEVEDO, LMA., RIZZI, PEV., BEZERRA-NETO, JF. and ROLLA, ME. 2005. Origens e efeitos do aporte externo de nutrientes em um reservatório tropical de grande porte: Reservatório de São Simão (MG, GO). In NOGUEIRA, MG., HENRY, R. and JORCIN, A. (Orgs.) Ecologia de reservatórios. São Carlos, SP: RiMa. p. 127-164.

SHANNON, CE. and WEAVER, W. 1949. The mathematical theory of communication. Illinois, Urbana, USA: Ed. John Wiley \& Sons. $125 \mathrm{p}$

SHAPIRO, J. 1973. Blue-green algae: Why they become dominant. Sci. NY, vol. 179, no. 4, p. 382-384.

SMITH, V. 1983. Low nitrogen to phosphorus ratios favor dominance by blue green algae in Lake phytoplankton. Sci. NY, vol. 221 , no. 8 , p. 669-671.

TRAIN, S., JATI, S., RODRIGUES, LC. and PIVATO, BM. 2005. Distribuição espacial e temporal do fitoplâncton em três reservatórios da bacia do Paraná. In RODRIGUES, L., THOMAZ, SM., AGOSTINHO, AA. and GOMES, LC. (Orgs.). Biocenose em reservatórios - padrões espaciais e temporais. São Paulo, SP: Ed. RIMA. p. 73-85.

TUCCI, A. and SANT'ANNA, CL. 2003. Cylindrospermopsis raciborskii (Woloszynska) Seenayya \& Subba raju (cyanobacteria): variação semanal e relações com fatores ambientais em um reservatório eutrófico, São Paulo, SP, Brasil. Revista Brasil. Bot., vol. 26, no. 1, p. 97-112.

TUNDISI, JG., MATSUMARA-TUNDISI, T. and ROCHA, O. 1999. Theoretical basis for reservoir managemente. In TUNDISI, JG. and STRAKRABA, M. (Eds.). Theoretical reservoir ecology and its applications. São Carlos: International Institute of Ecology - IIE.

WEHR, JD. and SHEATH, RG. 2003. Freshwater Algae of North America: Ecology and Classification. Lond: Academic Press. $918 \mathrm{p}$ 